\title{
Pseudo-homogeneous 1D RANS radial model for heat transfer in tubular packed beds
}

\author{
I. Thiagalingam ${ }^{\mathrm{a}, \mathrm{b}, *}$, P. Sagaut ${ }^{\mathrm{c}}$ \\ a Sorbonne Universités, UPMC Univ Paris 06, CNRS, UMR 7190, Institut Jean Le Rond \\ d'Alembert, F-75005 Paris, France \\ ${ }^{b}$ Paris Saclay Research Center-Air Liquide, 1 Chemin de la porte des Loges, Les loges en \\ Josas, BP 126, 78354 Jouy-en-Josas, France \\ ${ }^{c}$ Aix-Marseille Université, CNRS, Centrale Marseille, M2P2 UMR 7340, 13451 \\ Marseille, France
}

\begin{abstract}
A RANS zonal pseudo-homogeneous 1D radial heat transfer model is derived using an homogenization technique along with high-fidelity microscopic simulation to calibrate the model free parameters. Thus, it is brought to light the importance of the mechanical dispersion in the mixing process, the similarity between turbulent and dispersive dynamics, the existence of a near wall zone characterized by a channeling effect which is responsible for the thermal resistance over the zone. A linear law for the effective thermal conductivity is proposed to assess the heat transfer within the disrupted thermal boundary layer. The model showed its ability to estimate the effective conductivity and the temperature field in the radial direction with satisfaction. Very good agreements are also found in the near wall zone where the temperature gradients are the highest. The model well estimated also the value of the wall temperature and the wall heat transfer coefficient for an imposed heat flux at the wall.
\end{abstract}

Keywords: Packed bed, turbulence, wall temperature, dispersion, porous medium, up-scaling, CFD, heat transfer, boundary layer

\section{Introduction}

Packed beds with low tube-to-particle diameters ratio are widely used in chemical engineering, e.g. in steam methane reforming processes for Hydrogen production. The heat transfer problem is intensively investigated in such

\footnotetext{
${ }^{*}$ Corresponding author

Email address: ilango.thiagalingam@gmail.com (I. Thiagalingam)
} 
$5 \quad$ systems as the local temperature drives directly the efficiency of the catalytic reactions occurring on the solid particles' surface. The accurate prediction of the radial temperature profile from the tube wall to the middle of the bed is an important step towards the reliable assessment of reaction rates along the packed bed. Another critical point justifying the development of radial heat transfer models is the wall temperature assessment. Indeed, the near wall region faces strong thermal resistance as shown by the high temperature gradient profiles over this region [1. Accurate heat control is then required near the wall as in the operating conditions a difference of $20 \mathrm{~K}$ at the tube wall can divide its life time by two or more.

For high ratio between tube and particles diameters $(N \gg 10)$, the radial packing configuration is almost homogeneous as the wall induced inhomogeneity is confined in the very near vicinity of the tube. Hence, one can assume that both the axial velocity $U_{z}$ and the effective thermal conductivity $\lambda_{r}$ are constant in the radial direction and the extra near wall resistance can be lumped in a wall heat transfer coefficient $h_{w}$. These are the main hypotheses of the extensively used classical pseudo-homogeneous two dimensional plug flow heat transfer model commonly referred to as the $\lambda_{r}-h_{w}$ model [2].

However, discrepancies between literature correlations for $\lambda_{r}[3]$ and namely 25 for $h_{w}$ [4] highlight that the assumed hypotheses have no more validity for relatively low tube-particle diameters ratio $(N<10)$ packed beds. Moreover, experimental measurements [5] and recent CFD simulations of fixed bed reactors [6] highlight an oscillating profile of the radial void which smoothly decreases with the distance from the wall. However, near wall profile displays a steep decrease from the wall (where the void is equal to one) up to a distance of $d_{p} / 2$, where $d_{p}$ is the particle diameter. The velocity profile which is linked to the porosity profile displays the same features [6, 7]. The wall heat transfer coefficient which is a sort of a boundary condition is no more suitable for an extended wall zone (its width is not negligible compared to the bulk zone). For a comprehensive review on radial heat transfer problem, see [8].

An alternative model to the $\lambda_{r}-h_{w}$ model [9, 10, 11, 12, 13, which is able to dispense with the apparent wall heat transfer coefficient $h_{w}$ has been first proposed by [14]. It consists of deriving a bed effective conductivity which 40 depends on the radial position. It was thus highlighted that the effective thermal conductivity $\lambda_{r}(r)$ is sharply damped in the vicinity of the wall [15]. From this observation, numerous two-layer models were developed [16, 17, 18, 19, 13. to distinguish the near wall zone heat transfers from the bulk ones. However, deep insights into the physical mechanisms were lacking among 45 those models. Then, Borkink et al. [20] bring to light the existing link 
between the near wall channeling effect and the thermal resistance showing that the near wall particular packing configuration is responsible for the conductivity damping in the vicinity of the wall. More realistic models were then proposed by authors 21. For instance, Lerou et al. and Marivoet et al. 22, 23] resolved the energy conservation equation with a void profile depending on the radial position and a velocity profile calculated with the Brinkman-Darcy-Forchheimer (BDF, [24]) model. It was shown that models tacking into account both the radial inhomogeneity and the flow dynamic improve significantly the temperature profile prediction [12, 25].

The aim of this paper is to derive a new pseudo-homogeneous $1 \mathrm{D}$ radial model for the heat transfer in low-N packed beds, using the high-fidelity microscopic CFD solutions. The main features of the present model are the physical insights brought to the modeling of the near wall heat transfer mechanisms. The porous medium framework is used to up-scale unequivocally pore scale relevant data obtained via high-fidelity $3 \mathrm{D}$ simulations to the reactor scale. This is practically achieved extending the concept of Representative Elementary Volume (REV) to anisotropic wall bounded porous medium [26]. Afterwards, turbulent flow governing pseudo-homogeneous equations including mechanical dispersion are derived and validated against 3D detailed numerical simulations. Then, the flow features are used to describe the thermal mixing and derive the radial effective convective conductivity called for the 1D radial pseudo-homogeneous heat transfer model development. Both wall temperature and the bed temperature profile are finally validated over the reference data obtained performing $3 \mathrm{D}$ fine simulations at the pore scale.

2. 3D high-fidelity numerical simulations of turbulent flow and heat transfers at the pore scale in packed beds and data upscaling

\subsection{Packing/meshing step}

A tubular random and periodic packing configuration with spheres is considered (Fig,2). The ratio between tube and particle diameters (respectively $d_{t}$ and $\left.d_{p}\right)$ is taken equal to $10\left(N=d_{t} / d_{p}=10\right)$. The domain is composed of 835 particles and the average porosity is equal to $\phi_{\text {moy }}=0.443$ (see Table 1). The packing is generated thanks to the commercial algorithm DigiPac ${ }^{T M}$ which has demonstrated its capability to generate reliable and realistic tubular packings mainly for spherical particles [27, 28, 29, 30]. The packing is considered to be homogeneous in the axial (Fig, 3) and azimuthal directions but presenting radial inhomogeneity (Fig 4). 
During the mesh generation step, one has to face high skewed cells generated in the regions surrounding particle-particle or particle-tube contact points. Moreover, the distribution of pore size is quite large as far as random packings are concerned. Thus, very narrow gaps can also be found which is another source of low quality cells. To preclude convergence problems in CFD simulations, one can slightly modify the packing configuration [31, 32, 33, 34, 6, 35, 36]. Local modifications of the geometry are recommended against global modifications. Indeed, global modifications can lead errors on porosity and so on the pressure drop of $4 \%$ and $12-15 \%$ respectively [37]. The strategy employed here [26] consists in first detecting particles one close to another with a distance less than $1 \%$ of $d_{p}$. For those particles, radius is then increased by $2 \%$ of $d_{p}$. The overlapping part are removed and the increased particles take then back their original size. A minimal gap between particles is thus guaranteed (Fig.1). The same approach is applied for the particle-tube contact points or narrow gaps. The tube radius is decreased by $2 \%$ of $d_{p}$ and parts of particles laying outside of the new cylinder are removed. The tube takes then back its original dimensions.

\subsection{Computational Fluid Dynamics (CFD) model setup}

The fluid domain is meshed with tetrahedral elements and numerical simulations are performed with the commercial solver ANSYS Fluent 13.0. Periodic boundary conditions are set at the inlet/outlet faces imposing a mass flow. Particles are assumed to be adiabatic and a constant heat flux is set on the tube wall. The Reynolds Averaged Navier-Stokes and energy equations are solved to get flow and temperature fields in the fluid domain. The turbulence dynamic is described with the two-equation $k-\epsilon$ model. The enhanced wall treatment which combines a two-layer model with an enhanced wall law is set to capture the near wall dynamic: when the mesh is fine enough $\left(y^{+} \approx 1\right)$, the viscous affected region is solved by the two-layer model and when the mesh is coarse, a wall law is rather used (see ANSYS Documentation for further details). The near wall treatment used here is much more suited for complex wall flow encountered in packed beds than a standard wall law. Indeed, the $y^{+}$values are spread over a wide range as they depend directly on both the local mesh size and the local flow configuration (recirculating, squeezed, downward, accelerated or stagnant flow).

As for the numerical discretization, the first order upwind scheme is used to solve momentum, energy and turbulence equations. The standard scheme is used to solve the pressure and the pressure-velocity coupling is realized with the SIMPLE scheme. 


\begin{tabular}{|l|c|c|c|}
\hline Particle Shape & Number of particles & N & $\phi_{\text {moy }}$ \\
\hline \hline Spherical & 835 & 10 & 0.443 \\
\hline
\end{tabular}

Table 1: Main characteristics of the present packed bed.

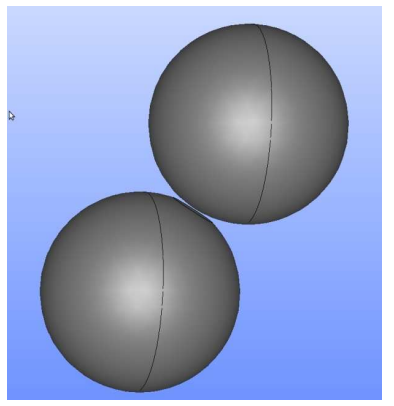

Figure 1: Contact points locally handled by creating a small gap between particles.

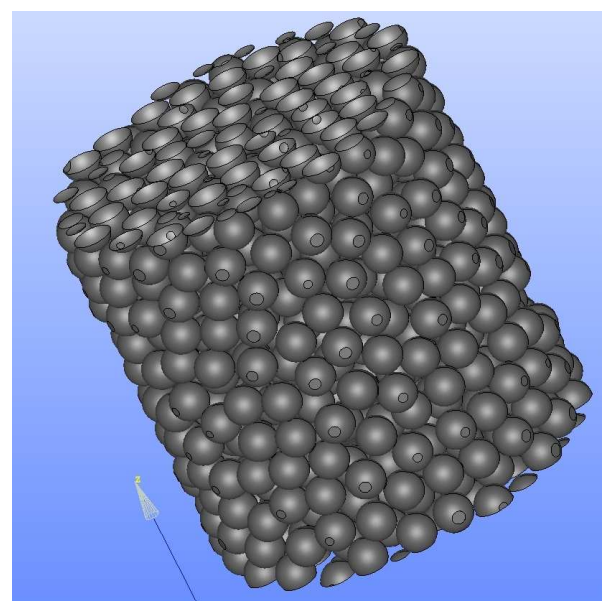

Figure 2: Periodic random packing of spherical particles.

\section{3. upscaling}

In order to derive macroscopic governing equations for a turbulent flow in porous media, both time and spatial averaging operators are applied to instantaneous mass, momentum and energy conservation equations. The averaging operators split instantaneous quantity into two parts: the mean 


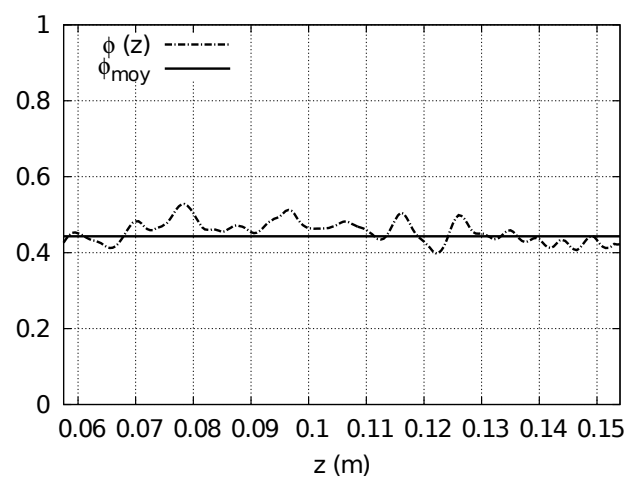

Figure 3: Axial porosity.

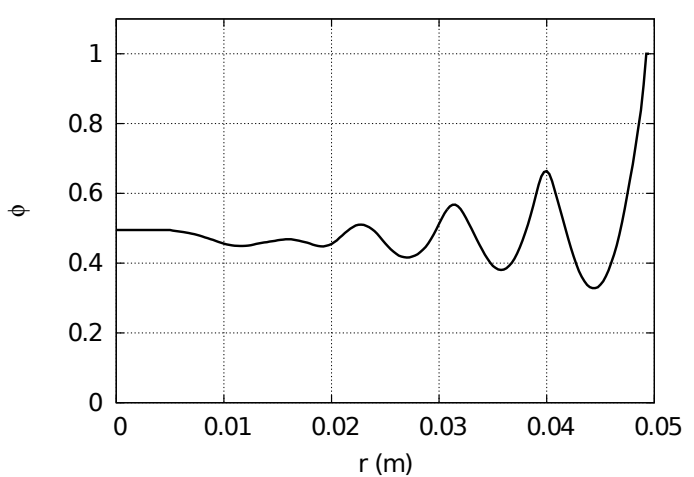

Figure 4: Radial porosity.

quantity and its fluctuation [38]. Hence the time decomposition reads,

$$
\varphi=\bar{\varphi}+\varphi^{\prime}
$$

where $\bar{\varphi}=\frac{1}{\Delta t} \int_{t}^{t+\Delta_{t}} \varphi d t$ is the time averaged quantity and $\varphi^{\prime}$ the time fluctuation around the mean quantity. Following the same reasoning for the spatial averaging operator, one can write

$$
\varphi=\langle\varphi\rangle^{i}+\delta \varphi
$$

where $\langle\varphi\rangle^{i}$ is the intrinsic average of $\varphi$ in the fluid and $\delta \varphi$ the spatial deviation. According to the scale separation's assumption between microscopic and macroscopic quantities, one can obtain $\langle\delta \varphi\rangle^{i}=\bar{\varphi}^{\prime}=0$. The volume averaged quantity reads,

$$
\langle\varphi\rangle^{v}=\frac{1}{V} \int_{V} \varphi d V
$$

where $V$ is a volume in which the average is carried out. It is also referred to as the REV, the Representative Elementary Volume [39, 40]. A methodology to define the appropriate REV for wall bounded medium presenting radial heterogeneity has been developed in [26] and is used in the present study to upscale unequivocally pore scale data to the observation scale (bed scale). When one uses the right REV for volume averaging, it can be showed [38] that the two averaging operators defined above permute $\langle\bar{\varphi}\rangle^{i}=\overline{\langle\varphi\rangle^{i}}$. When the porous medium is saturated by the fluid, one can also link the intrinsic average and the volume average by the following expression :

$$
\langle\varphi\rangle^{v}=\phi\langle\varphi\rangle^{i}
$$


where $\phi=\frac{V_{f}}{V}$, denotes the porosity. While applying the space averaging operators to flow governing equations for instance, one needs to permute derivatives and averaging operators. It is showed from [41,

$$
\langle\nabla \varphi\rangle^{v}=\nabla\langle\varphi\rangle^{v}+\frac{1}{V} \int_{A} \boldsymbol{n} \varphi \mathrm{dA},
$$

with $d A$ an element of solid surface, $\boldsymbol{n}$ the normal vector directed from the solid phase toward the fluid phase and $A$ the solid surface.

Applying the volume averaging operator to the momentum and energy conversation equations at the pore scale one gets, after some algebra, at the macroscopic scale:

$$
\begin{gathered}
\nabla_{j}\left(\phi\left\langle\bar{u}_{j}\right\rangle^{i}\right)=0 \\
\nabla_{j}[-\phi\left\langle\bar{u}_{j}\right\rangle^{i}\left\langle\bar{u}_{i}\right\rangle^{i}+\nu \nabla_{j}\left[\phi\left\langle\bar{u}_{i}\right\rangle^{i}\right]-\delta_{i j} \phi \frac{\langle\bar{P}\rangle^{i}}{\rho} \underbrace{-\phi\left\langle R_{i j}\right\rangle^{i}}_{\text {turbulent diffusion }} \underbrace{\left.-\phi\left\langle\delta \bar{u}_{i} \delta \bar{u}_{j}\right\rangle^{i}\right]}_{\text {dispersion }} \\
+\underbrace{\frac{\nu}{V} \int \nabla_{j} \bar{u}_{i} n_{j} d S-\frac{1}{V \rho} \int P n_{i} d S}_{\text {viscous and pressure drag }}=0
\end{gathered}
$$

where $\overline{u_{i}^{\prime} u_{j}^{\prime}}=R_{i j}$.

$$
\begin{array}{r}
0=\left(\rho C_{p}\right)_{f} \nabla_{i}[-\phi\left\langle\bar{u}_{i}\right\rangle^{i}\langle\bar{T}\rangle^{i} \underbrace{-\phi\left\langle\delta \bar{u}_{i} \delta \bar{T}\right\rangle^{i}}_{\text {dispersion }} \underbrace{-\phi\left\langle\overline{u_{i}^{\prime} T^{\prime}}\right\rangle^{i}}_{\text {turbulent diffusion }}] \\
+\nabla_{i}[\left(\lambda_{f} \phi\right) \nabla_{i}\langle\bar{T}\rangle^{i}+\underbrace{\frac{1}{V} \int n_{i} \lambda_{f} \delta \bar{T}_{f} d s}_{\text {tortuosity }}]
\end{array}
$$

Both the second order moments and surface terms need a closure and those sub-filter terms can be assessed carrying out simulations within the REV [42, 43].

Mesh convergence study is finally achieved for REV-averaged quantities. Radial profiles of a certain number of the REV-averaged quantities are depicted on Figs. 5, 6, 7, 8, 9, 10 for different mesh densities. One can observe that a satisfactory convergence is obtained on all quantities of interest for the present study. 


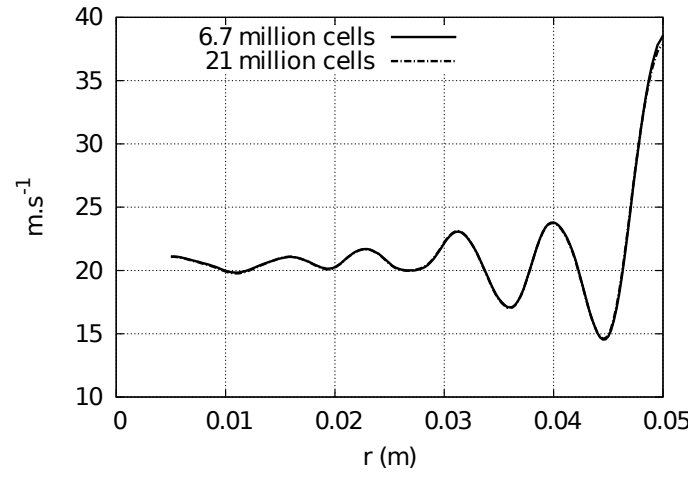

Figure 5: Radial profile of $\left\langle\bar{u}_{z}\right\rangle^{i}$ for different mesh densities.

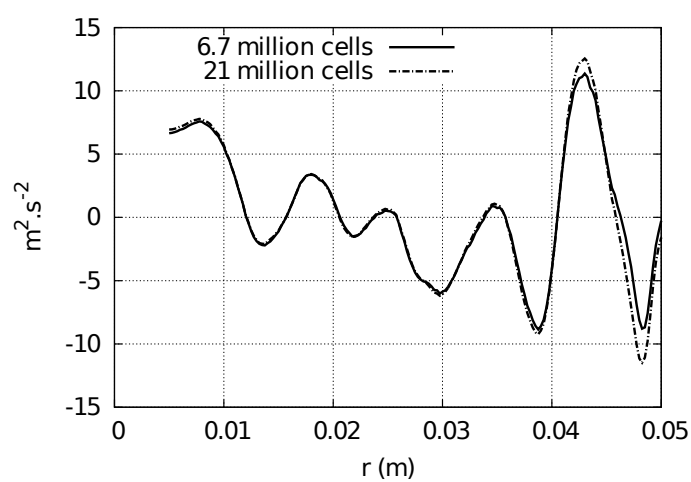

Figure 7: Radial profile of $\left\langle\delta \bar{u}_{z} \delta \bar{u}_{r}\right\rangle^{i}$ for different mesh densities.

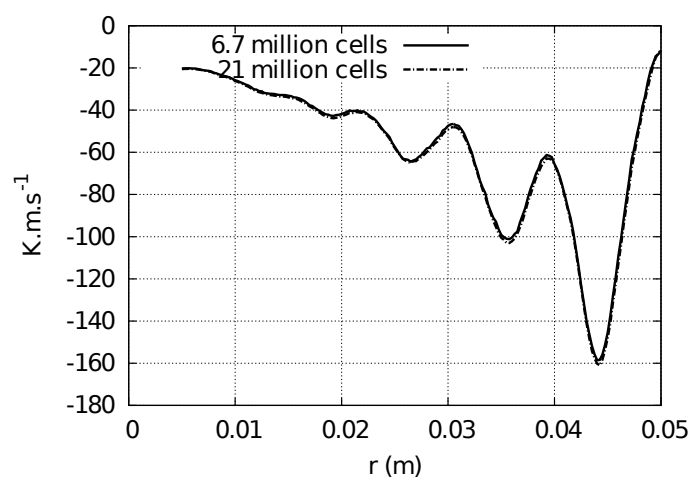

Figure 9: Radial profile of $\left\langle\delta \bar{u}_{z} \delta \bar{T}\right\rangle^{i}$ for different mesh densities.

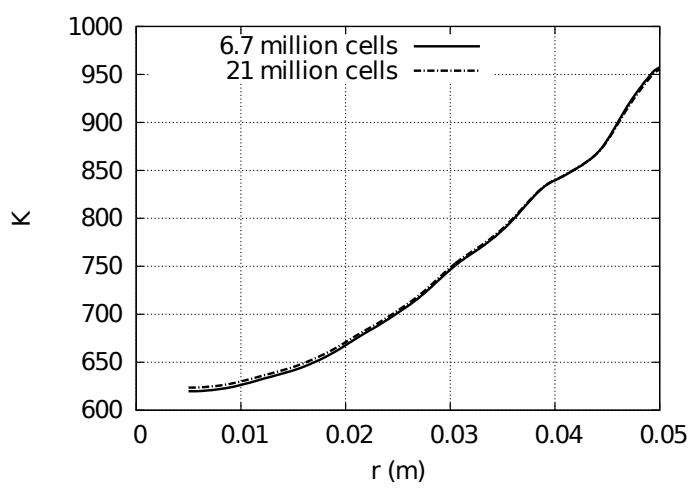

Figure 6: Radial profile of $\langle\bar{T}\rangle^{i}$ for different mesh densities.

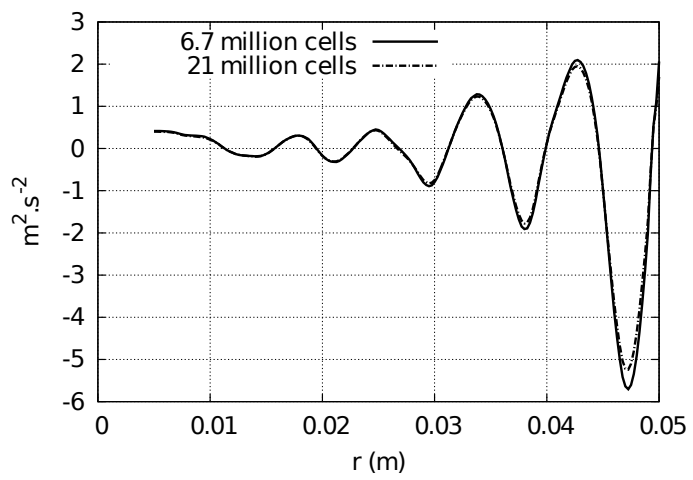

Figure 8: Radial profile of $\left\langle\overline{\left\langle u_{z}^{\prime} u_{r}^{\prime}\right.}\right\rangle^{i}$ for different mesh densities.

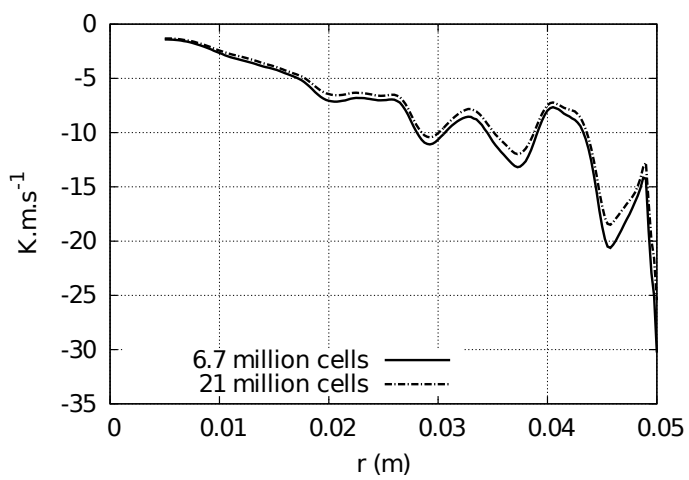

Figure 10: Radial profile of $\left\langle\overline{T^{\prime} u_{r}^{\prime}}\right\rangle^{i}$ for different mesh densities. 


\section{Macroscopic model for turbulent flow dynamic in packed beds}

\subsection{Turbulence in porous medium}

Turbulence structures were observed by numerous experimental studies [44, 45, 46, 47] as soon as the Reynolds number based on pore size was above a few hundreds. The experimentally observed deviation of the flow dynamic to the Darcy law was thus attributed to the development of local turbulence [48. Turbulence models were then developed at the macroscopic scale by applying volume averaging operator to Reynolds averaged NavierStokes equations (RANS) [42, 49, 50, 38.

A new concept was recently introduced by Teruel et al. [51, 52]. It consists in not distinguishing time fluctuations from the spatial ones but in considering fluctuations as the difference between the instantaneous quantity and the double averaged (time and space) quantity.

$$
\psi(\boldsymbol{r}, t)=\overline{\bar{\psi}}(\boldsymbol{x}, t)+\psi^{\prime \prime}(\boldsymbol{r}, \boldsymbol{x}, t)
$$

with

$$
\overline{\bar{\psi}}(\boldsymbol{x}, t)=\frac{1}{\Delta t} \int_{\Delta t}\left[\frac{1}{V_{f}} \int_{V_{f}} \psi d V\right] d \tau=\frac{1}{V_{f}} \int_{V_{f}}\left[\frac{1}{\Delta t} \int_{\Delta t} \psi d \tau\right] d V
$$

and

$$
\overline{\bar{\psi}}^{\prime \prime}(\boldsymbol{r}, \boldsymbol{x}, t) \cong 0, \quad \overline{\overline{\bar{\psi}}}(\boldsymbol{x}, t) \cong \overline{\bar{\psi}}(\boldsymbol{x}, t)
$$

Thus, the total turbulent kinetic energy at the macroscopic scale becomes,

$$
\begin{aligned}
k_{\text {Teruel }} & =\frac{\overline{\overline{u_{j}^{\prime \prime} u_{j}^{\prime \prime}}}}{2} \\
& =\frac{\left\langle\overline{u_{j}^{\prime} u_{j}^{\prime}}\right\rangle^{i}}{2}+\frac{\left\langle\delta \bar{u}_{j} \delta \bar{u}_{j}\right\rangle^{i}}{2} \\
& =\langle k\rangle^{i}+\frac{\left\langle\delta \bar{u}_{j} \delta \bar{u}_{j}\right\rangle^{i}}{2}
\end{aligned}
$$

It is made up of a turbulent part $\langle k\rangle^{i}$ and a dispersive part $\left\langle k_{d}\right\rangle^{i}=\frac{\left\langle\delta \bar{u}_{j} \delta \bar{u}_{j}\right\rangle^{i}}{2}$. The dispersion can be significant [53] and its contribution to the total kinetic energy is even above $60-70 \%$ 21] as far as packed beds are concerned (Figs. 11 and 12 . 


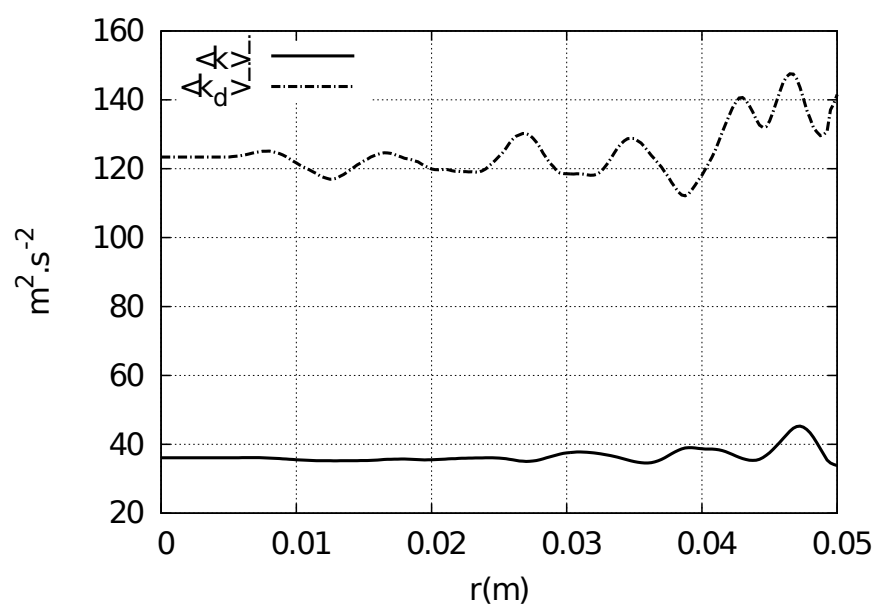

Figure 11: Radial profiles of the REV-averaged turbulent $\langle k\rangle^{i}$ and dispersive $\left\langle k_{d}\right\rangle^{i}$ kinetic energies in packed beds.

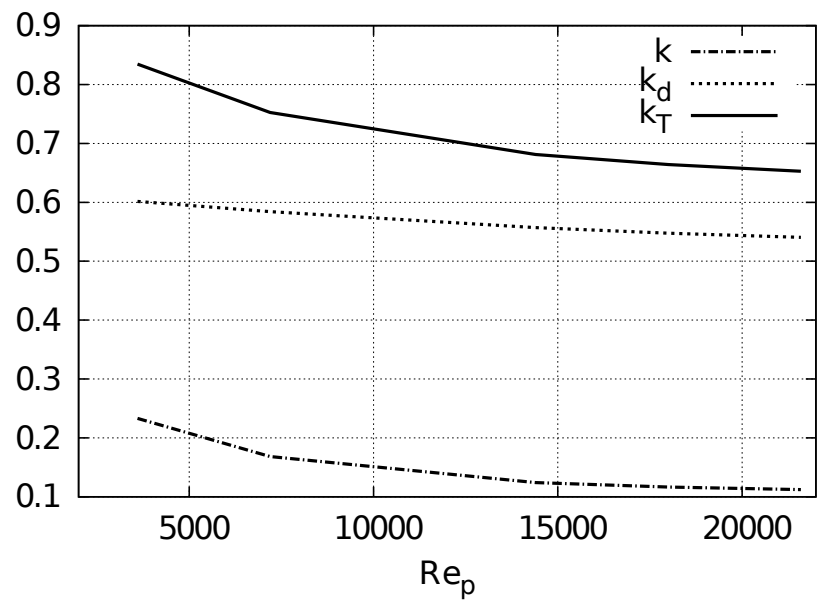

Figure 12: Evolution of the contribution of different kinetic energies with the Reynolds number. $\quad k=\frac{1}{R} \int_{0}^{R} d r \frac{\phi\langle k\rangle^{i}}{U_{s}^{2}}, \quad k_{d}=\frac{1}{R} \int_{0}^{R} d r \frac{\phi\left\langle k_{d}\right\rangle^{i}}{U_{s}^{2}}$ et $k_{T}=$ $\frac{1}{R} \int_{0}^{R} d r \frac{\phi\left[\langle k\rangle^{i}+\left\langle k_{d}\right\rangle^{i}\right]}{U_{s}^{2}} . U_{s}$ is the superficial velocity.

\subsection{Macroscopic $\langle K\rangle^{i}-\langle\epsilon\rangle^{i}$ model}

Following Teruel et al., a two equation model is derived at the macroscopic scale to describe the total turbulence. The total kinetic energy is defined as

$$
\langle K\rangle^{i}=\langle k\rangle^{i}+\left\langle k_{d}\right\rangle^{i}
$$


where $\langle k\rangle^{i}$ and $\left\langle k_{d}\right\rangle^{i}$ describe respectively the time and spatial fluctuations at the macroscopic scale. The equation governing the total kinetic energy is obtained as follows: First, the volume averaging operator is applied to the turbulent kinetic energy at the pore scale to up-scale it at the macroscopic scale (see Appendix A). The governing equation for the dispersive part is then obtained (see Appendix B) [54]. They are finally summed up to get the governing equation for the total fluctuations.

$$
\begin{aligned}
0 & =-\nabla_{j}\left[\phi\left\langle\overline{u_{j}}\right\rangle^{i}\langle K\rangle^{i}\right]+\nu \nabla_{j}^{2}\left[\phi\langle K\rangle^{i}\right]-\phi\left[\left\langle R_{i j}\right\rangle^{i}+\left\langle\delta \overline{u_{i}} \delta \overline{u_{j}}\right\rangle^{i}\right] \nabla_{j}\left\langle\overline{u_{i}}\right\rangle^{i} \\
& -\nabla_{j}\left(\phi\left[\left\langle\frac{u_{j}^{\prime}}{\rho}+\overline{u_{i}^{\prime} u_{i}^{\prime} u_{j}^{\prime}}\right\rangle^{i}+\frac{1}{\rho}\left\langle\delta \overline{u_{j}} \delta \bar{P}\right\rangle^{i}+\left\langle\delta \overline{u_{i}} \delta \overline{u_{i}} \delta \overline{u_{j}}\right\rangle^{i}\right]\right)-\phi\langle\epsilon\rangle^{i}-\phi\left\langle\epsilon_{d}\right\rangle^{i} \\
& -\nabla_{j}\left[\phi\left\langle\delta \bar{u}_{j} \delta k\right\rangle^{i}\right]+\frac{\nu}{V} \int \nabla_{j} k n_{j} d S+\left\langle\overline{u_{j}}\right\rangle^{i} \nabla_{j} \phi\left[3\left\langle k_{d}\right\rangle^{i}-\frac{\left\langle\overline{u_{i}}\right\rangle^{i 2}}{2}\right] \\
& +\frac{\left\langle\overline{u_{i}}\right\rangle^{i}}{\rho V} \int \delta \bar{P} n_{i} d S-\frac{\nu\left\langle\overline{u_{i}}\right\rangle^{i}}{V} \int \nabla_{j} \delta \overline{u_{i}} n_{j} d S \\
& -\nabla_{j}\left[\phi\left\langle\delta \overline{u_{i}} \delta R_{i j}\right\rangle^{i}\right]-\nu \nabla_{j}\left[\nabla_{j} \phi \frac{\left\langle\overline{u_{i}}\right\rangle^{i 2}}{2}\right]+\phi\left\langle\delta \overline{u_{i}} \delta \overline{u_{j}} \nabla_{j} \delta \overline{u_{i}}\right\rangle^{i}
\end{aligned}
$$

One can recognize on the right hand side the convection term, the molecular diffusion, the production term, the dynamic (turbulent and dispersive) diffusion and the energy destruction term, respectively. The other terms stem from the averaging operations and the radial inhomogeneity.

For configurations under consideration, one can show that the dispersive part of the dissipation rate $\left(\left\langle\epsilon_{d}\right\rangle^{i}=\nu\left\langle\frac{\partial \delta \bar{u}_{j}}{\partial x_{j}} \frac{\partial \delta \bar{u}_{j}}{\partial x_{j}}\right\rangle^{i}\right)$ is negligible (Fig. 13. Thus, $\left\langle\epsilon_{T}\right\rangle^{i} \approx\langle\epsilon\rangle^{i}$.

The governing equation for the total dissipation rate becomes,

$$
\begin{aligned}
& 0=-\nabla_{j}\left(\phi\langle\epsilon\rangle^{i}\left\langle\overline{u_{j}}\right\rangle^{i}\right)+\nabla_{j}\left[\left(\nu+\frac{\left\langle\nu_{t}\right\rangle^{i}}{\sigma_{\epsilon}}\right) \nabla_{j}\left(\phi\langle\epsilon\rangle^{i}\right)\right]-\frac{C_{1}}{\langle k\rangle^{i}}\left\langle R_{i j}\right\rangle^{i} \nabla_{j}\left(\phi\left\langle\bar{u}_{i}\right\rangle^{i}\right)\langle\epsilon\rangle^{i} \\
& -\frac{C_{2} \phi}{\langle k\rangle^{i}}\langle\epsilon\rangle^{i 2}-\nabla_{j}\left(\phi\left\langle\delta \epsilon \delta \overline{u_{j}}\right\rangle^{i}\right)+\nabla_{j}\left(\frac{\left(\nu+\left\langle\nu_{t}\right\rangle^{i} / \sigma_{\epsilon}\right)}{V} \int \epsilon n_{j} d S\right) \\
& +\frac{1}{V} \int \nu\left(\nabla_{j} \epsilon\right) n_{j} d S+\frac{1}{\sigma_{\epsilon}} \nabla_{j}\left(\phi\left\langle\delta \nu_{t} \nabla_{j} \delta \epsilon\right\rangle^{i}\right)-\frac{C_{1} \phi}{\langle k\rangle^{i}}\left(\left\langle\delta R_{i j} \nabla_{j} \delta \overline{u_{i}}\right\rangle^{i}\langle\epsilon\rangle^{i}\right. \\
& \left.+\left\langle\delta \epsilon \delta R_{i j}\right\rangle^{i} \nabla_{j}\left\langle\overline{u_{i}}\right\rangle^{i}+\left\langle R_{i j}\right\rangle^{i}\left\langle\delta \epsilon\left(\nabla_{j} \delta \overline{u_{i}}\right)\right\rangle^{i}+\left\langle\delta R_{i j} \delta \epsilon\left(\nabla_{j} \delta \overline{u_{i}}\right)\right\rangle^{i}\right)-\frac{C_{2}}{\langle k\rangle^{i}} \phi\langle\delta \epsilon \delta \epsilon\rangle^{i}
\end{aligned}
$$




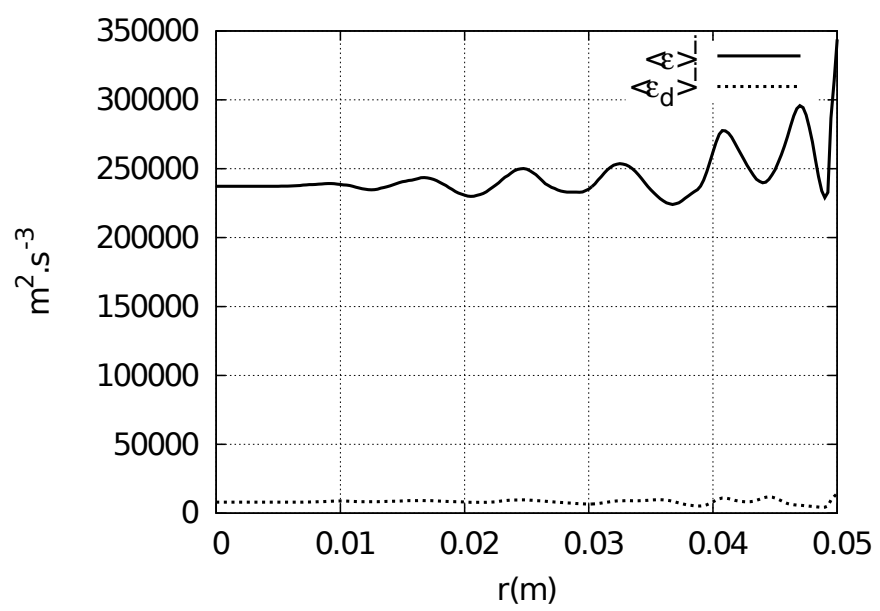

Figure 13: Radial profile of the turbulent $\left(\langle\epsilon\rangle^{i}\right)$ an dispersive $\left(\left\langle\epsilon_{d}\right\rangle^{i}\right)$ part of the total dissipation rate at the macroscopic scale.

\subsection{Closure relations}

Considering a fully developed flow which enables to neglect the axial macroscopic gradient $\left(\frac{\partial\langle\cdot\rangle^{i}}{\partial z}\right),(7)$ can be rewritten in cylindrical coordinates,

$$
\begin{aligned}
0 & =-\frac{\rho}{r} \nabla_{r}\left[r \phi\left\langle\bar{u}_{r}\right\rangle^{i}\left\langle\bar{u}_{z}\right\rangle^{i}\right]-\nabla_{z}\left[\phi\langle\bar{P}\rangle^{i}\right]-\frac{\rho}{r} \nabla_{r}\left[r \phi\left\langle R_{z r}\right\rangle^{i}+r \phi\left\langle\delta \overline{u_{r}} \delta \overline{u_{z}}\right\rangle^{i}\right] \\
& +\mu \frac{1}{r} \nabla_{r}\left[r \nabla_{r}\left(\phi\left\langle\bar{u}_{z}\right\rangle^{i}\right)\right]+\frac{\mu}{V} \int \nabla_{i} \bar{u}_{z} n_{i} d S-\frac{1}{V} \int P n_{z} d S
\end{aligned}
$$

with $i=z, r$. It can be numerically verified that the second order moments appearing in the macroscopic momentum conservation equation $(16)$ can be linked to the velocity gradient thanks to a dynamic viscosity (Fig. 14).

$$
-\phi\left[\left\langle R_{z r}\right\rangle^{i}+\left\langle\delta \overline{u_{r}} \delta \overline{u_{z}}\right\rangle^{i}\right]=\left\langle\nu_{T}\right\rangle^{i} \nabla_{r}\left[\phi\left\langle\bar{u}_{z}\right\rangle^{i}\right]
$$

where the dynamic viscosity reads,

$$
\begin{aligned}
& \left\langle\nu_{T}\right\rangle^{i}=0.09 \frac{\langle K\rangle^{i 2}}{\langle\epsilon\rangle^{i}} \times\left[\frac{1}{2}\left(1-\tanh \left[20\left(r-\left(R-d_{p} / 2\right)\right) / d_{p}\right]\right) \times C_{m 1}\right. \\
& \left.+\frac{1}{2}\left(1+\tanh \left[20\left(r-\left(R-d_{p} / 2\right)\right) / d_{p}\right]\right) \times C_{m 2}\right]
\end{aligned}
$$

$\langle K\rangle^{i}$ is the total kinetic energy. It is worth noting that a near wall zone is identified with a thickness of $d_{p} / 2$. The dynamic viscosity is lower in the vicinity of the wall than in the bulk zone and this property is captured thanks to the coefficients $C_{m 1}$ and $C_{m 2}$. 


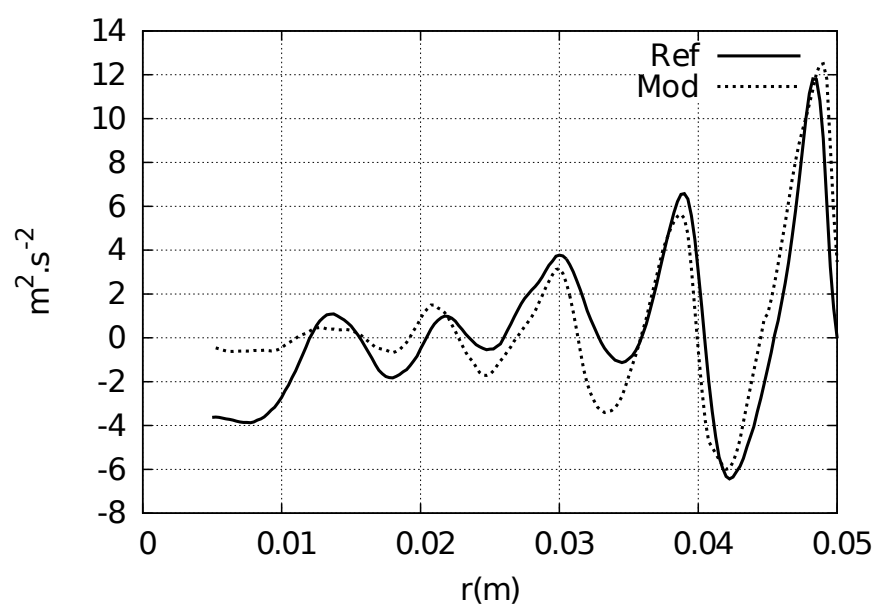

Figure 14: Radial profile of the second order moments (left hand side of (17)) denoted as "Ref" and the model based on the Boussinesq hypothesis (right hand side of (17)) denoted as "Mod". They are computed via 3D numerical simulations at the pore scale at $R e_{p} \approx 7800\left(R e_{p}\right.$ is the Reynolds number based on the particle diameter and the superficial velocity).

The surface terms is then closed with a modified two-zonal Ergun law [55].

$$
\begin{aligned}
& \frac{\mu}{V} \int \nabla_{i} \bar{u}_{z} n_{i} d S-\frac{1}{V} \int P n_{z} d S=-\left[\frac{1}{2}\left(1-\tanh \left[20\left(r-\left(R-d_{p} / 2\right)\right) / d_{p}\right]\right) C_{F 1}\right. \\
& \left.+\frac{1}{2}\left(1+\tanh \left[20\left(r-\left(R-d_{p} / 2\right)\right) / d_{p}\right]\right) C_{F 2}\right] \times \rho \frac{(1-\phi)^{0.1}}{d_{p} \phi^{5}}\left[\phi\left\langle\bar{u}_{z}\right\rangle^{i}\right]^{2}
\end{aligned}
$$

In the following, the macroscopic equations are written in their superficial forms $(\psi)_{s}=\phi\langle\bar{\psi}\rangle^{i}$. Thus, the macroscopic Navier-Stokes equations reads,

$$
\begin{gathered}
\nabla_{z}\left[\left(u_{z}\right)_{s}\right]=0 \\
0=-\nabla_{z}(P)_{s}+\frac{1}{r} \nabla_{r}\left[r\left\{\mu+\rho\left(\nu_{T}\right)_{s}\right\} \nabla_{r}\left(u_{z}\right)_{s}\right]-h\left(\phi, d_{p}\right)\left(u_{z}\right)_{s}^{2}
\end{gathered}
$$

with

$$
\begin{aligned}
& \left(\nu_{T}\right)_{s}=0.09 \frac{(K)_{s}^{2}}{(\epsilon)_{s}} \times\left[\frac{1}{2}\left(1-\tanh \left[20\left(r-\left(R-d_{p} / 2\right)\right) / d_{p}\right]\right) \frac{C_{m 1}}{\phi}\right. \\
& \left.+\frac{1}{2}\left(1+\tanh \left[20\left(r-\left(R-d_{p} / 2\right)\right) / d_{p}\right]\right) \frac{C_{m 2}}{\phi}\right]
\end{aligned}
$$


and

$$
\begin{aligned}
h\left(\phi, d_{p}\right) & =\rho \frac{(1-\phi)^{0.1}}{d_{p} \phi^{5}} \times\left[\frac{1}{2}\left(1-\tanh \left[20\left(r-\left(R-d_{p} / 2\right)\right) / d_{p}\right]\right) C_{F 1}\right. \\
& \left.+\frac{1}{2}\left(1+\tanh \left[20\left(r-\left(R-d_{p} / 2\right)\right) / d_{p}\right]\right) C_{F 2}\right]
\end{aligned}
$$

The fully developed total kinetic energy equation in the cylindrical coordinates reads,

$$
\begin{aligned}
& 0=\frac{1}{r} \nabla_{r}\left(r \nu \nabla_{r}\left[\phi\langle K\rangle^{i}\right]\right)-\phi\left[\left\langle R_{z r}\right\rangle^{i}+\left\langle\delta \overline{u_{z}} \delta \overline{u_{r}}\right\rangle^{i}\right] \nabla_{r}\left\langle\bar{u}_{z}\right\rangle^{i} \\
& -\frac{1}{r} \nabla_{r}\left(r \phi\left[\left\langle\frac{\overline{P^{\prime} u_{r}^{\prime}}}{\rho}+\overline{u_{i}^{\prime} u_{i}^{\prime} u_{r}^{\prime}}\right\rangle^{i}-\frac{1}{\rho}\left\langle\delta \overline{u_{r}} \delta \bar{P}\right\rangle^{i}-\left\langle\delta \overline{u_{i}} \delta \overline{u_{i}} \delta \overline{u_{r}}\right\rangle^{i}\right]\right)-\phi\langle\epsilon\rangle^{i} \\
& -\frac{1}{r} \nabla_{r}\left[r \phi\left\langle\delta \bar{u}_{r} \delta k\right\rangle^{i}\right]+\frac{\nu}{V} \int \nabla_{i} k n_{i} d S \\
& +\frac{\left\langle\overline{u_{z}}\right\rangle^{i}}{\rho V} \int \delta \bar{P} n_{z} d S-\frac{\nu\left\langle\overline{u_{z}}\right\rangle^{i}}{V} \int \nabla_{i} \delta \overline{u_{z}} n_{i} d S \\
& -\frac{1}{r} \nabla_{r}\left[r \phi\left\langle\delta \overline{u_{i}} \delta R_{i r}\right\rangle^{i}\right]-\nu \frac{1}{r} \nabla_{r}\left[r \nabla_{r} \phi \frac{\left\langle\overline{u_{z}}\right\rangle^{i 2}}{2}\right]+\phi\left\langle\delta \overline{u_{i}} \delta \overline{u_{j}} \nabla_{j} \delta \overline{u_{i}}\right\rangle^{i}
\end{aligned}
$$

with $i, j=z, r, \theta$. The production term is rewritten as,

$$
\begin{aligned}
& -\phi\left[\left\langle R_{i r}\right\rangle^{i}+\left\langle\delta \overline{u_{i}} \delta \overline{u_{r}}\right\rangle^{i}\right] \nabla_{r}\left\langle\overline{u_{i}}\right\rangle^{i}=-\phi\left[\left\langle R_{z r}\right\rangle^{i}+\left\langle\delta \overline{u_{z}} \delta \overline{u_{r}}\right\rangle^{i}\right] \nabla_{r}\left[\phi\left\langle\overline{u_{z}}\right\rangle^{i}\right] \\
& -\phi\left[\left\langle R_{z r}\right\rangle^{i}+\left\langle\delta \overline{u_{z}} \delta \overline{u_{r}}\right\rangle^{i}\right] \times\left[\frac{1-\phi}{\phi} \nabla_{r}\left[\phi\left\langle\overline{u_{z}}\right\rangle^{i}\right]-\frac{\nabla_{r} \phi}{\phi}\left\langle\overline{u_{z}}\right\rangle^{i}\right] \\
& =\left(\nu_{T}\right)_{s}\left[\nabla_{r}\left(u_{z}\right)_{s}\right]^{2}+\left(\nu_{T}\right)_{s}\left[\nabla_{r}\left(u_{z}\right)_{s}\right] \times\left[\frac{1-\phi}{\phi} \nabla_{r}\left(u_{z}\right)_{s}-\frac{\nabla_{r} \phi}{\phi^{2}}\left(u_{z}\right)_{s}\right]
\end{aligned}
$$

and the flux terms can be clustered as

$$
\begin{aligned}
& -\left(\phi\left[\left\langle\frac{\overline{P^{\prime} u_{r}^{\prime}}}{\rho}+\overline{u_{i}^{\prime} u_{i}^{\prime} u_{r}^{\prime}}\right\rangle^{i}-\frac{1}{\rho}\left\langle\delta \overline{u_{r}} \delta \bar{P}\right\rangle^{i}-\left\langle\delta \overline{u_{i}} \delta \overline{u_{i}} \delta \overline{u_{r}}\right\rangle^{i}+\left\langle\delta \overline{u_{r}} \delta k\right\rangle^{i}+\left\langle\delta \overline{u_{i}} \delta R_{i r}\right\rangle^{i}\right]\right. \\
& \left.+\nu \nabla_{r} \phi \frac{\left\langle\overline{u_{z}}\right\rangle^{i 2}}{2}\right)=\frac{\left(\nu_{T}\right)_{s}}{\sigma_{K}}\left[\nabla_{r}(K)_{s}\right]
\end{aligned}
$$

where the macroscopic turbulent Prandtl number $\sigma_{K}$ is to be determined. The remaining terms are finally clustered as an effective production which includes sub-filter production and the inhomogeneous part of the macroscopic 
production.

$$
\begin{aligned}
& \frac{\nu}{V} \int \nabla_{i} k n_{i} d S+\frac{\left\langle\overline{u_{z}}\right\rangle^{i}}{\rho V} \int \delta \bar{P} n_{z} d S-\frac{\nu\left\langle\overline{u_{z}}\right\rangle^{i}}{V} \int \nabla_{i} \delta \overline{u_{z}} n_{i} d S+\phi\left\langle\delta \overline{u_{i}} \delta \overline{u_{j}} \nabla_{j} \delta \overline{u_{i}}\right\rangle^{i} \\
& +\left(\nu_{T}\right)_{s}\left[\nabla_{r}\left(u_{z}\right)_{s}\right] \times\left[\frac{1-\phi}{\phi} \nabla_{r}\left(u_{z}\right)_{s}-\frac{\nabla_{r} \phi}{\phi^{2}}\left(u_{z}\right)_{s}\right]=f(\phi)\left(u_{z}\right)_{s}^{3}
\end{aligned}
$$

where $f(\phi)$ is a function to be determined. The macroscopic governing equation for the total kinetic energy reads finally,

$$
0=\frac{1}{r} \nabla_{r}\left[r\left(\nu+\frac{\left(\nu_{T}\right)_{s}}{\sigma_{K}}\right) \nabla_{r}(K)_{s}\right]+\left(\nu_{T}\right)_{s}\left[\nabla_{r}\left(u_{z}\right)_{s}\right]^{2}+f(\phi)\left(u_{z}\right)_{s}^{3}-(\epsilon)_{s}
$$

The macroscopic dissipation rate equation becomes in cylindrical coordinates,

$$
\begin{aligned}
& 0=\frac{1}{r} \nabla_{r}\left[r\left(\nu+\frac{\left\langle\nu_{t}\right\rangle^{i}}{\sigma_{\epsilon}}\right) \nabla_{r}\left(\phi\langle\epsilon\rangle^{i}\right)\right]-\frac{C_{1}}{\langle k\rangle^{i}}\left\langle R_{z r}\right\rangle^{i} \nabla_{r}\left(\phi\left\langle\overline{u_{z}}\right\rangle^{i}\right)\langle\epsilon\rangle^{i}-\frac{C_{2} \phi}{\langle k\rangle^{i}}\langle\epsilon\rangle^{i 2} \\
& -\frac{1}{r} \nabla_{r}\left[r \phi\left\langle\delta \epsilon \delta \overline{u_{r}}\right\rangle^{i}\right]+\frac{1}{r} \nabla_{r}\left(r \frac{\left(\nu+\left\langle\nu_{t}\right\rangle^{i} / \sigma_{\epsilon}\right)}{V} \int \epsilon n_{r} d S\right)+\frac{1}{V} \int \nu\left(\nabla_{i} \epsilon\right) n_{i} d S \\
& +\frac{1}{r \sigma_{\epsilon}} \nabla_{r}\left[r \phi\left\langle\delta \nu_{t} \nabla_{r} \delta \epsilon\right\rangle^{i}\right]-\frac{C_{1} \phi}{\langle k\rangle^{i}}\left(\left\langle\delta R_{i j} \nabla_{i} \delta \overline{u_{j}}\right\rangle^{i}\langle\epsilon\rangle^{i}+\left\langle\delta \epsilon \delta R_{z r}\right\rangle^{i} \nabla_{r}\left\langle\overline{u_{z}}\right\rangle^{i}\right. \\
& \left.+\left\langle R_{i r}\right\rangle^{i}\left\langle\delta \epsilon\left(\nabla_{r} \delta \overline{u_{i}}+\nabla_{i} \delta \overline{u_{r}}\right)\right\rangle^{i}+\left\langle\delta R_{i j} \delta \epsilon \nabla_{j} \delta \overline{u_{i}}\right\rangle^{i}\right)-\frac{C_{2}}{\langle k\rangle^{i}} \phi\langle\delta \epsilon \delta \epsilon\rangle^{i}
\end{aligned}
$$

with $i, j=z, r, \theta$. the production term can be rewritten as

$$
\begin{aligned}
& -\frac{C_{1}}{\langle k\rangle^{i}}\left\langle R_{z r}\right\rangle^{i} \nabla_{r}\left(\phi\left\langle\overline{u_{z}}\right\rangle^{i}\right)\langle\epsilon\rangle^{i} \approx-\phi\left[\left\langle R_{z r}\right\rangle^{i}+\left\langle\delta \overline{u_{z}} \delta \overline{u_{r}}\right\rangle^{i}\right] \nabla_{r}\left[\phi\left\langle\overline{u_{z}}\right\rangle^{i}\right] \frac{C_{\epsilon 1}}{\langle K\rangle^{i}}\langle\epsilon\rangle^{i} \\
& +\phi\left\langle\delta \overline{u_{z}} \delta \overline{u_{r}}\right\rangle^{i} \nabla_{r}\left[\phi\left\langle\overline{u_{z}}\right\rangle^{i}\right] \frac{C_{\epsilon 1}}{\langle K\rangle^{i}}\langle\epsilon\rangle^{i}
\end{aligned}
$$

The overall fluxes are clustered as

$$
\begin{aligned}
& \left(\frac{\left\langle\nu_{t}\right\rangle^{i}}{\sigma_{\epsilon}} \nabla_{r}\left(\phi\langle\epsilon\rangle^{i}\right)-\phi\left\langle\delta \epsilon \delta \overline{u_{r}}\right\rangle^{i}+\frac{\left(\nu+\left\langle\nu_{t}\right\rangle^{i} / \sigma_{\epsilon}\right)}{V} \int \epsilon n_{r} d S\right. \\
& \left.+\frac{1}{\sigma_{\epsilon}} \phi\left\langle\delta \nu_{t} \nabla_{r} \delta \epsilon\right\rangle^{i}\right)=\frac{\left(\nu_{T}\right)_{s}}{\sigma_{\epsilon K}}\left[\nabla_{r}(\epsilon)_{s}\right]
\end{aligned}
$$


where $\sigma_{\epsilon K}$ is the Prandtl number associated to the macroscopic dissipation rate. The destruction term becomes,

$$
-\frac{C_{2} \phi}{\langle k\rangle^{i}}\langle\epsilon\rangle^{i 2}-\frac{C_{2}}{\langle k\rangle^{i}} \phi\langle\delta \epsilon \delta \epsilon\rangle^{i} \approx-C_{\epsilon 2} \frac{(\epsilon)_{s}^{2}}{(K)_{s}}
$$

The remaining terms are once again clustered as an effective production

$$
\begin{aligned}
& \frac{1}{V} \int \nu\left(\nabla_{i} \epsilon\right) n_{i} d S-\frac{C_{1} \phi}{\langle k\rangle^{i}}\left(\left\langle\delta R_{i j} \nabla_{i} \delta \overline{u_{j}}\right\rangle^{i}\langle\epsilon\rangle^{i}+\left\langle\delta \epsilon \delta R_{i r}\right\rangle^{i} \nabla_{r}\left\langle\overline{u_{i}}\right\rangle^{i}\right. \\
& \left.+\left\langle R_{i r}\right\rangle^{i}\left\langle\delta \epsilon\left(\nabla_{r} \delta \overline{u_{i}}+\nabla_{i} \delta \overline{u_{r}}\right)\right\rangle^{i}+\left\langle\delta R_{i j} \delta \epsilon \nabla_{j} \delta \overline{u_{i}}\right\rangle^{i}\right) \\
& +\phi\left\langle\delta \overline{u_{z}} \delta \overline{u_{r}}\right\rangle^{i} \nabla_{r}\left[\phi\left\langle\overline{u_{z}}\right\rangle^{i}\right] \frac{C_{\epsilon 1}}{\langle K\rangle^{i}}\langle\epsilon\rangle^{i}=g\left(\phi, d_{p}\right)\left(u_{z}\right)_{s}^{4}
\end{aligned}
$$

The governing equation describing the macroscopic dissipation rate reads then in its superficial form,

$$
\begin{aligned}
& 0=\frac{1}{r} \nabla_{r}\left[r\left(\nu+\frac{\left(\nu_{T}\right)_{s}}{\sigma_{\epsilon K}}\right) \nabla_{r}(\epsilon)_{s}\right]+C_{\epsilon 1} \frac{(\epsilon)_{s}}{(K)_{s}}\left(\nu_{T}\right)_{s}\left[\nabla_{r}\left(u_{z}\right)_{s}\right]^{2} \\
& +g\left(\phi, d_{p}\right)\left(u_{z}\right)_{s}^{4}-C_{\epsilon 2} \frac{(\epsilon)_{s}^{2}}{(K)_{s}}
\end{aligned}
$$

with $C_{\epsilon 1}$ and $C_{\epsilon 2}$ model coefficients to be determined.

\section{4. model validation}

The following expression is suggested to represent the radial void profile at the macroscopic scale (Fig, 15).

$\phi^{*}\left(r^{\star}\right)= \begin{cases}1 & \text { si } r^{\star}=0 \\ 0.465\left[1+1.1 \exp \left(-48 r^{\star 2}\right)\right]+0.16 \exp \left(-0.2 r^{\star 2}\right) \sin \left(2.46 \pi r^{\star}\right) & \text { sinon }\end{cases}$

with $r^{\star}=(R-r) / d_{p}$.

Both the model coefficients and functions are obtained by optimizing the matching with the reference data. Hence, $C_{m 1}=0.444, C_{m 2}=0.05, \sigma_{K}=1$, $\sigma_{\epsilon K}=1.3, C_{\epsilon 1}=1.44, C_{\epsilon 2}=1.92$. Then,

$$
\begin{gathered}
C_{F 1}\left(R e_{p}\right)= \begin{cases}0.26 R e_{p}^{-0.14}-0.02 & 4000 \leq R e_{p} \leq 19500 \\
0.046 & 19500 \leq R e_{p} \leq 31000\end{cases} \\
C_{F 2}\left(R e_{p}\right)= \begin{cases}5.5 \times 10^{-6} R e_{p}+0.2 & 4000 \leq R e_{p} \leq 19500 \\
0.1 & 19500 \leq R e_{p} \leq 31000\end{cases}
\end{gathered}
$$




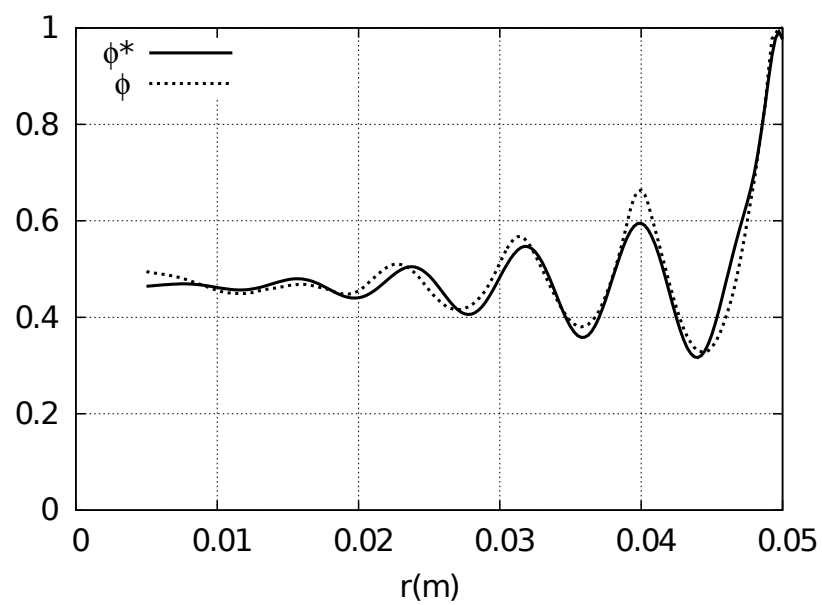

Figure 15: Validation of the suggested expression for the porosity profile $\left(\phi^{*}\right)$ against the porosity characterizing the reference system $(\phi)$.

$$
\begin{aligned}
f\left(\phi^{*}\right) & =\rho C_{K 1}\left(R e_{p}\right) \frac{\left(1-\phi^{*}\right)}{\phi^{* 2}} \times \frac{1}{2}\left(1-\tanh \left[20\left(r-\left(R-d_{p} / 2\right)\right) / d_{p}\right]\right) \\
& +\left[\rho C_{K 2}\left(R e_{p}\right) \phi^{* 5}+\rho C_{K 3}\left(R e_{p}\right) \frac{\left(1-\phi^{*}\right)}{\phi^{* 4}} \exp \left(-4\left(r-\left(R-d_{p} / 2\right)\right) / d_{p}\right)\right] \\
& \times \frac{1}{2}\left(1+\tanh \left[20\left(r-\left(R-d_{p} / 2\right)\right) / d_{p}\right]\right)
\end{aligned}
$$

with,

$$
\begin{gathered}
C_{K 1}\left(R e_{p}\right)= \begin{cases}-0.78 R e_{p}^{0.5}+132 & 4000 \leq R e_{p} \leq 15600 \\
38.41 & 15600 \leq R e_{p} \leq 31000\end{cases} \\
C_{K 2}\left(R e_{p}\right)= \begin{cases}-8 \times 10^{-4} R e_{p}+20 & 4000 \leq R e_{p} \leq 19600 \\
5.6 & 19600 \leq R e_{p} \leq 31000\end{cases}
\end{gathered}
$$

and

$$
C_{K 3}\left(R e_{p}\right)= \begin{cases}\left(5 \times 10^{-6} R e_{p}\right)^{-1}+12 & 4000 \leq R e_{p} \leq 19600 \\ 23.11 & 19600 \leq R e_{p} \leq 31000\end{cases}
$$


Finally,

$$
\begin{aligned}
g\left(\phi^{*}, d_{p}\right) & =\rho C_{E 1}\left(R e_{p}\right) \frac{\left(1-\phi^{*}\right)^{0.5}}{d_{p}^{2} \phi^{* 5}} \times \frac{1}{2}\left(1-\tanh \left[20\left(r-\left(R-d_{p} / 2\right)\right) / d_{p}\right]\right) \\
& +\rho C_{E 2}\left(R e_{p}\right) \frac{\left(1-\phi^{*}\right)^{0.4}}{d_{p}^{2} \phi^{* 8}} \times \frac{1}{2}\left(1+\tanh \left[20\left(r-\left(R-d_{p} / 2\right)\right) / d_{p}\right]\right)
\end{aligned}
$$

with

$$
C_{E 1}\left(R e_{p}\right)= \begin{cases}-0.049 R e_{p}^{0.26}+0.66 & 4000 \leq R e_{p} \leq 15600 \\ 0.069 & 1560 \leq R e_{p} \leq 31000\end{cases}
$$

and

$$
C_{E 2}\left(R e_{p}\right)= \begin{cases}\left(0.01 R e_{p}\right)^{-0.8} & 4000 \leq R e_{p} \leq 19600 \\ 0.016 & 19600 \leq R e_{p} \leq 31000\end{cases}
$$

One has to notice that all those coefficients tend towards a constant value at high $R e_{p}$ (Fig. 16, 17, 18).

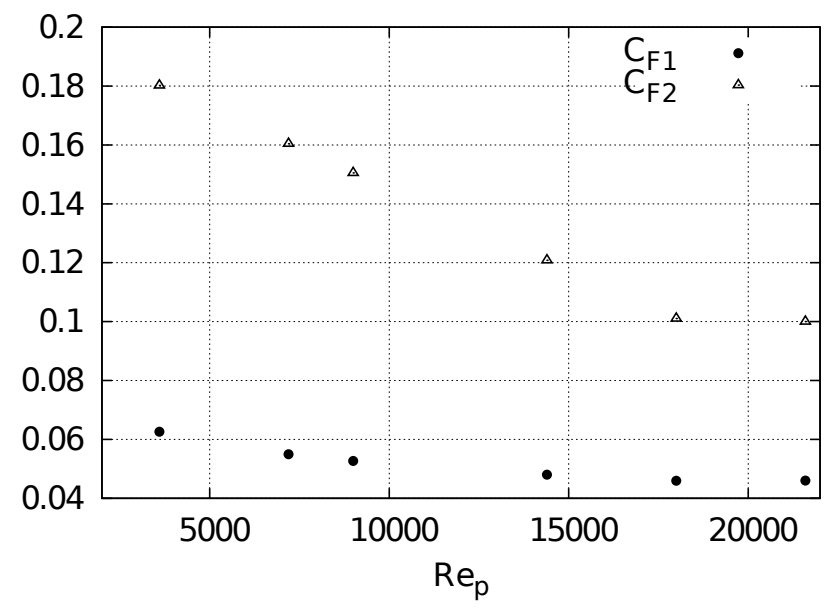

Figure 16: Reynolds dependence of $C_{F 1}$ and $C_{F 2}$.

In the following, superscript ${ }^{*}$ stands for quantities obtained performing macro-scale simulations. Pressure drops evaluated with 3D simulations at the pore scale and the ones assessed performing macroscopic simulations are reported on the Table 2 for a few chosen Reynolds numbers. Very good agreements are found between pore and bed scale simulations. Radial profiles of the mean velocity, the total turbulent kinetic energy and the macroscopic dissipation rate obtained performing macroscopic simulations are validated 


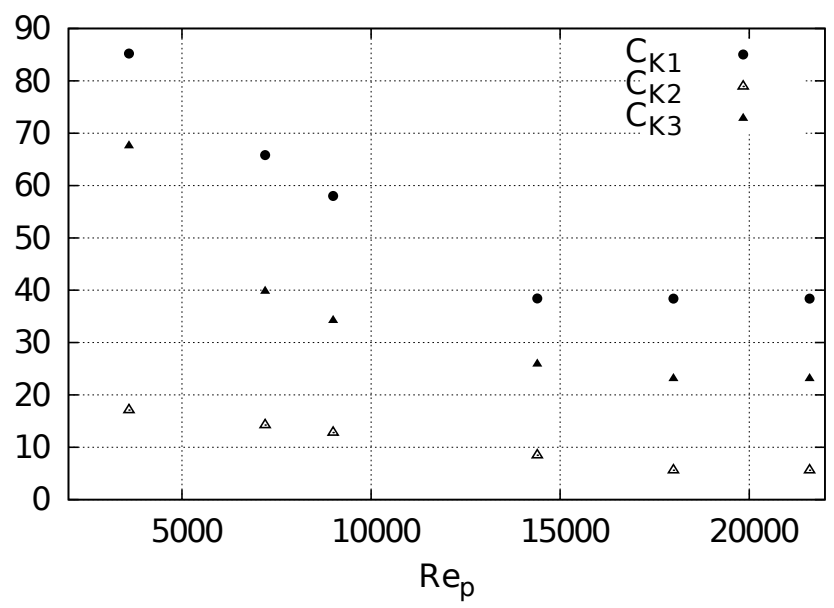

Figure 17: Reynolds dependence of $C_{K 1}, C_{K 2}$ and $C_{K 3}$.

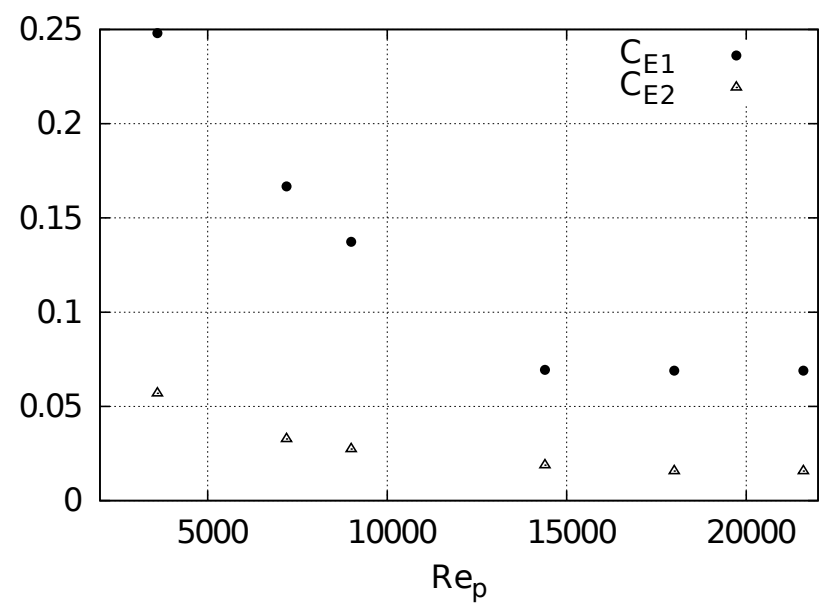

Figure 18: Reynolds dependence of $C_{E 1}$ and $C_{E 2}$

against the up-scaled microscopic ones (Figs. 19, 20, 21). Indeed, the oscillating profile and the near wall maximum are quite well recovered for all those quantities, even if the radial oscillations for the dissipation rate are not recovered by the macroscopic model. This shows that there exists local diffusion damping that should be taken into account by a radially varying Prandtl number associated to the dissipation rate, $\sigma_{\epsilon K}$. However, it is particularly worth noting that the near wall zone flow dynamic is well assessed. 


\begin{tabular}{|l|c|c|c|}
\hline$R e_{p}$ & $\Delta P / \Delta L($ Pascal $/ m)$ & $(\Delta P)^{*} / \Delta L($ Pascal $/ m)$ & Error \\
\hline \hline 2000 & -2272.8 & -2148.9 & $5.45 \%$ \\
4000 & -7841.1 & -7582.1 & $3.30 \%$ \\
7800 & -26668.3 & -26829.5 & $0.6 \%$ \\
15600 & -89880.4 & -91042.5 & $1.3 \%$ \\
19600 & -133767.5 & -130105.8 & $2.7 \%$ \\
23300 & -185831.1 & -186726.7 & $0.5 \%$ \\
\hline
\end{tabular}

Table 2: Pressure drop evaluation. $\operatorname{Micro}(\Delta P / \Delta L)$ vs. Macro $\left((\Delta P)^{*} / \Delta L\right)$

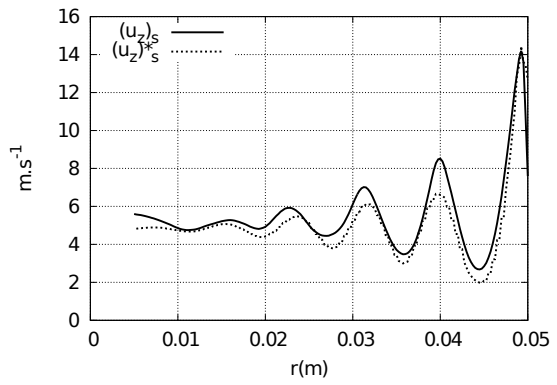

(a) $R e_{p}=4000$

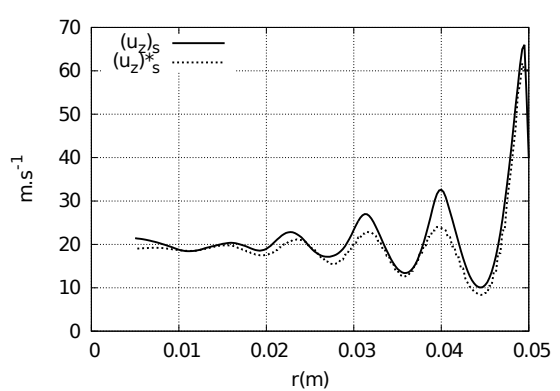

(c) $R e_{p}=15600$

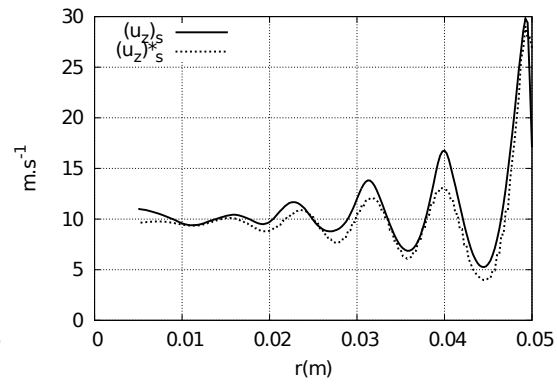

(b) $R e_{p}=7800$

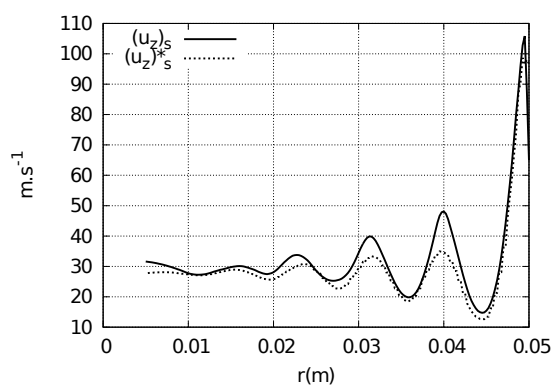

(d) $R e_{p}=23300$

Figure 19: Radial profile of the mean velocity, Micro $\left(u_{z}\right)_{s}$ vs. Macro $\left(u_{z}\right)_{s}^{*}$. 


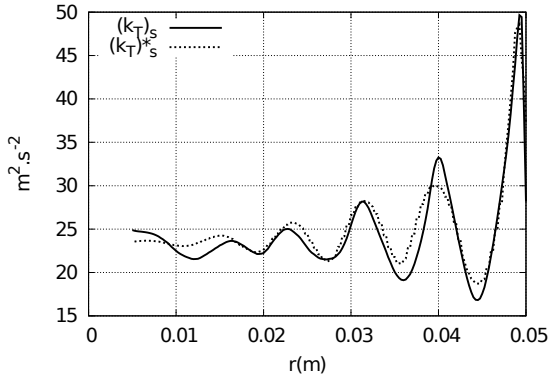

(a) $R e_{p}=4000$

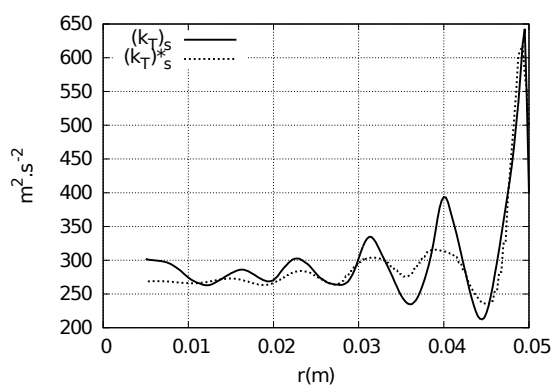

(c) $R e_{p}=15600$

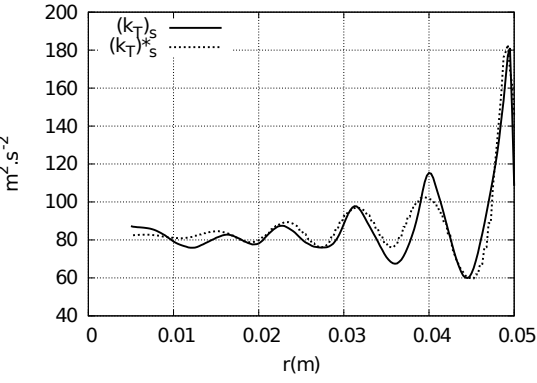

(b) $R e_{p}=7800$

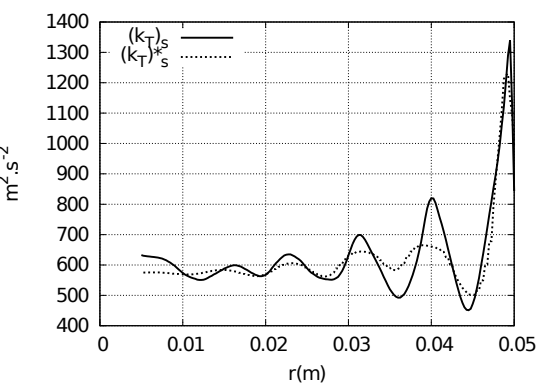

(d) $R e_{p}=23300$

Figure 20: Radial profile of the total kinetic energy, Micro $(k)_{s}$ vs. Macro $(k)_{s}^{*}$. 


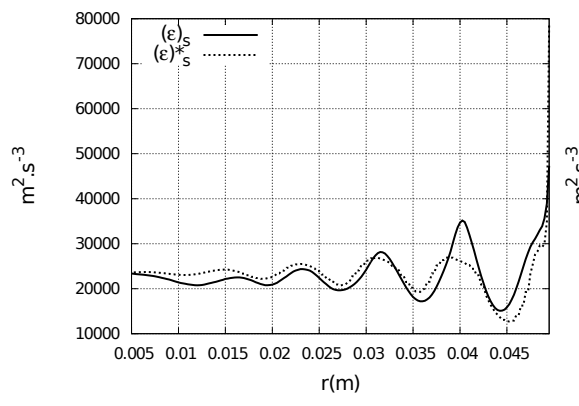

(a) $R e_{p}=4000$

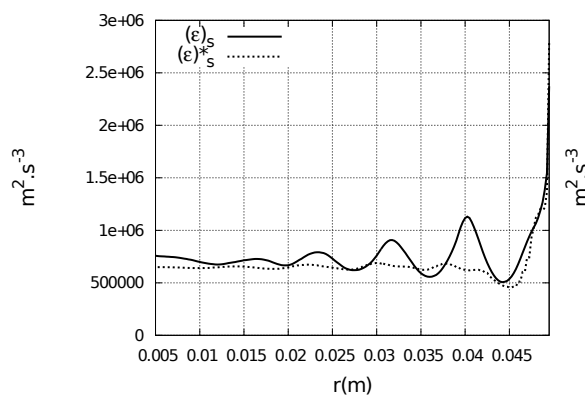

(c) $R e_{p}=15600$

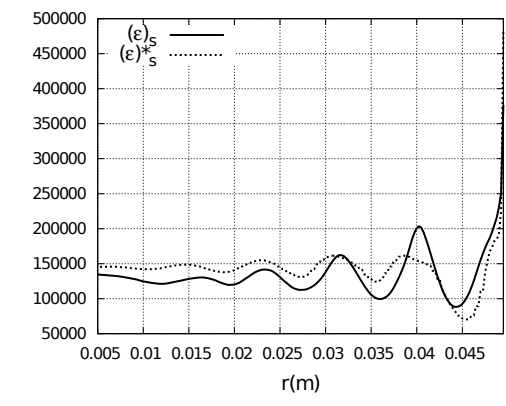

(b) $R e_{p}=7800$

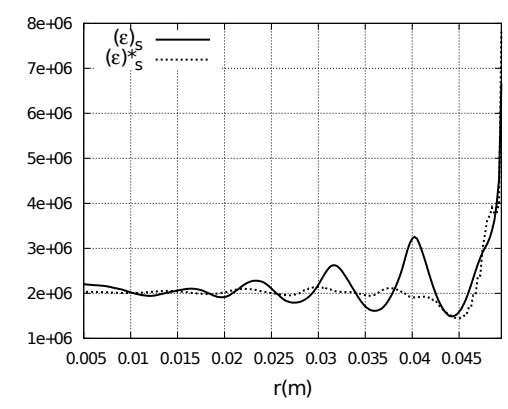

(d) $R e_{p}=23300$

Figure 21: Radial profile of the macroscopic dissipation rate, Micro $(\epsilon)_{s}$ vs. Macro $(\epsilon)_{s}^{*}$. 


\section{Pseudo-homogeneous 1D macroscopic radial model for heat trans- fer}

\subsection{Model of Mathey}

The macroscopic equation governing the heat transfer (8) can be rewritten in cylindrical coordinates as,

$$
\begin{aligned}
\left(\rho C_{p}\right)_{f} \phi\left\langle\bar{u}_{z}\right\rangle^{i} \frac{\partial}{\partial z}\langle\bar{T}\rangle^{i}= & -\left(\rho C_{p}\right)_{f} \frac{1}{r} \frac{\partial}{\partial r}\left[r \phi\left[\left\langle\overline{\delta u_{r} \delta T}\right\rangle^{i}+\left\langle\overline{u_{r}^{\prime} T^{\prime}}\right\rangle^{i}+\left\langle\bar{u}_{r}\right\rangle^{i}\langle\bar{T}\rangle^{i}\right]\right]+ \\
& \frac{1}{r} \frac{\partial}{\partial r} r\left[\left(\lambda_{f} \phi\right) \frac{\partial}{\partial r}\langle\bar{T}\rangle^{i}+\frac{1}{V} \int n_{r} \lambda_{f} \delta \bar{T}_{f} d s\right]
\end{aligned}
$$

As far as packed bed configurations are concerned, the surface term which represents the tortuosity can be neglected compared to the turbulent and dispersive fluxes (Fig 22).

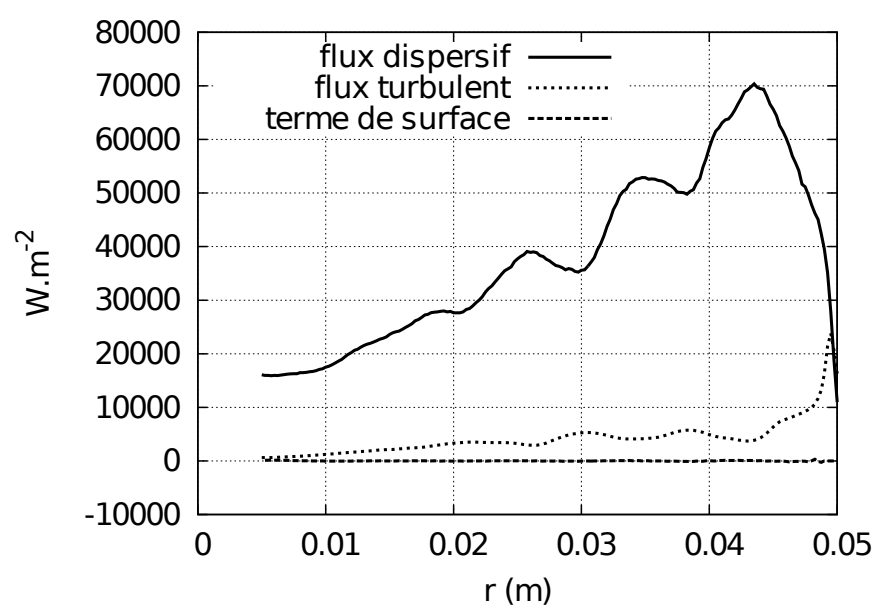

Figure 22: Radial profile of the tortuosity $\left(\frac{1}{V} \int n_{r} \lambda_{f} \delta \bar{T}_{f} d s\right)$, turbulent flux ( $\left.-\phi\left(\rho C_{p}\right)_{f}\left\langle\overline{u_{r}^{\prime} T^{\prime}}\right\rangle^{i}\right)$ and dispersive flux $\left(-\phi\left(\rho C_{p}\right)_{f}\left\langle\overline{\delta u_{r} \delta T}\right\rangle^{i}\right) . R e_{p}=14400$.

The concept of Teruel et al. [51, 52] which consists of clustering dispersive and turbulent fluctuations was extended by Mathey [21] to heat transfer problems. Indeed, the second order terms appearing in the energy conservation equation (45) were related to the temperature gradient through an effective conductivity depending on the radial position.

$$
-\left(\rho C_{p}\right)_{f} \phi\left[\left\langle\overline{\delta u_{r} \delta T}\right\rangle^{i}+\left\langle\overline{u_{r}^{\prime} T^{\prime}}\right\rangle^{i}\right]=\lambda_{e f f}(r) \frac{\partial}{\partial r}\langle\bar{T}\rangle^{i}
$$


with,

$$
\lambda_{e f f}=\phi\left(\rho C_{p}\right)_{f} \frac{\left\langle\nu_{T}\right\rangle^{i}}{\sigma_{t}}
$$

where $\sigma_{t}$ is the macroscopic Prandtl number. The dynamic viscosity is defined as

$$
\left\langle\nu_{T}\right\rangle^{i}=C_{\mu} \frac{\langle K\rangle^{i 2}}{\langle\epsilon\rangle^{i}}
$$

where $\langle K\rangle^{i}$ is the total kinetic energy (turbulence and dispersion are both included in it). With this approach, Mathey was able to derive a simple correlation for the medium effective conductivity [21],

$$
\begin{aligned}
\frac{\lambda_{e f f}(r)}{\lambda_{f}} & =\phi\left(\rho C_{p}\right)_{f} \frac{\left\langle\nu_{T}\right\rangle_{\infty}^{i}}{\lambda_{f} \sigma_{t}} \\
& =\frac{C_{k}^{2} C_{\mu}}{\sigma_{t} C_{\epsilon}} \frac{f_{k}^{2}(\phi(r))}{f_{\epsilon}(\phi(r))} \operatorname{Pr} \cdot R e_{p}
\end{aligned}
$$

with $\operatorname{Pr}$ the molecular Prandtl number, $R e_{p}$ the Reynolds number based on the superficial velocity and the particle diameter, $C_{k}$ and $C_{\epsilon}$ are model constants which represent respectively the strength of the kinetic energy subfilter production and the dissipation rate sub-filter production. $f_{\epsilon}(\phi(r))$ and $f_{k}(\phi(r))$ are functions depending on the porosity and damping to zero when getting closer to the wall. The radial profile of the effective conductivity was hence successfully estimated particularly in the bulk zone [56]. The model proposed by Mathey is improved here by tacking into account the radial inhomogeneity and the near wall zone heat transfer.

4.2. The bulk zone: $r<R-d_{p} / 2$

The effective convective conductivity in the radial direction is defined as

$$
\lambda_{e f f}(r)=-\rho_{f} \phi\left(C_{p}\right)_{f} \frac{\left\langle\overline{\delta u_{r} \delta T}\right\rangle^{i}+\left\langle\overline{u_{r}^{\prime} T^{\prime}}\right\rangle^{i}+\left\langle\bar{u}_{r}\right\rangle^{i}\langle\bar{T}\rangle^{i}}{\frac{\partial}{\partial r}\langle\bar{T}\rangle^{i}}
$$

The model proposed by Mathey [21] becomes,

$$
\left(\lambda_{e f f}\right)_{s}=\rho_{f}\left(C_{p}\right)_{f} \frac{\phi\left(\nu_{T}\right)_{s}}{\sigma_{t 1}\left(R e_{p}\right)}\left(1-a_{1}\left(R e_{p}\right) R \nabla_{r} \phi\right)
$$

with $\left(\nu_{T}\right)_{s}$ the dynamic viscosity defined in the previous section $\left(\nu_{T}\right)_{s}=$ $0.09 \frac{(K)_{s}^{2}}{(\epsilon)_{s}} \frac{C_{m 1}}{\phi}$ (see equation 18$)$ ), $\rho_{f}$ the fluid density, $\left(C_{p}\right)_{f}$ the fluid heat capacity, $\mathrm{R}$ the tube radius, $\sigma_{t 1}\left(R e_{p}\right)$ the macroscopic Prandtl number and $a_{1}\left(R e_{p}\right)$ a Reynolds depending constant measuring the inhomogeneity effect. 
The porosity gradient is related to the solid surface distribution within the REV according to the following expression [41]

$$
\nabla_{i} \phi=-\frac{1}{V} \int_{S} n_{i} d S
$$

When the medium is homogeneous $\left(-\frac{1}{V} \int_{S} n_{i} d S=0\right)$, one gets $\left(\lambda_{\text {eff }}\right)_{s}=$ $\rho_{f}\left(C_{p}\right)_{f} \frac{\phi\left(\nu_{T}\right)_{s}}{\sigma_{t 1 b}\left(R e_{p}\right)}$.

\subsection{The near wall zone: $R-d_{p} / 2 \leq r<R-d_{p} / 10$}

It was shown that the near wall zone is affected by a channeling effect. Indeed, the near wall dispersive mixing is reduced with the near wall void increase imposed by the packing configuration. Thus, even if the Reynolds number is locally increased, a significant part of the supplied heat at the wall is convected along the tube wall by the channeling effect. Therefore, the effective conductivity is weighted by the ratio between the total (turbulent and dispersive) mixing strength and the near wall channeling effect in order to assess the heat transfer efficiency in the near wall region.

$$
\left(\lambda_{e f f}\right)_{s}=\rho_{f}\left(C_{p}\right)_{f} \frac{\phi\left(\nu_{T}\right)_{s}}{\sigma_{t 2}\left(R e_{p}\right)}\left(1-a_{2}\left(R e_{p}\right) R \nabla_{r} \phi\right) \times\left(\frac{\sqrt{(K)_{s}}}{\left(u_{z}\right)_{s}}\right)^{\gamma}
$$

with $\gamma$ a constant.

\subsection{Heat transfer in the boundary layer: $R-d_{p} / 10 \leq r<R$}

In the viscous sublayer, the convective part is damped to zero and the effective conductivity is reduced to the molecular conductivity.

$$
\left(\lambda_{e f f}\right)_{s}=\phi \lambda_{f} \quad r^{+}<r_{L}^{+}
$$

with $\lambda_{f}$ the fluid conductivity, $r^{+}=(R-r) u_{\tau} / \nu$ wall distance expressed in wall unit and $r_{L}^{+}$the viscous sublayer thickness of the macroscopic model.

For the range of Reynolds numbers considered in the study (operating conditions), the boundary layer zone $r_{L}^{+} \nu / u_{\tau} \leq R-r<d_{p} / 10$ includes both the logarithmic and buffer zones. The macroscopic energy conservation equation over the boundary layer zone can be simplified as

$$
\frac{1}{r} \frac{\partial}{\partial r} r\left[\left(\phi \lambda_{e f f}\right) \frac{\partial}{\partial r}\langle\bar{T}\rangle^{i}\right]=A
$$

where $A$ a constant assessing the boundary layer overall heat transfer in the axial direction. 
We consider the case where the solid particles slightly disturb the thermal boundary layer. The temperature field can then be split up according to

$$
\langle\bar{T}\rangle^{i}=\langle\bar{T}\rangle^{i 0}+\epsilon\langle\bar{T}\rangle^{i 1}
$$

215 with $\epsilon<<1$.

0 order term in $\epsilon$ :

The temperature field is assumed to be logarithmic over the considered zone in the absence of solid particles. Thus,

$$
\begin{aligned}
& \frac{1}{d_{p}^{2}\left(R / d_{p}-r^{\star}\right)} \frac{\partial}{\partial r^{\star}}\left(R / d_{p}-r^{\star}\right)\left[\left(\phi \lambda_{e f f}\right) \frac{\partial}{\partial r^{\star}}\langle\bar{T}\rangle^{i 0}\right]=A \\
& \frac{\partial}{\partial r^{\star}}\left[\left(\phi \lambda_{e f f}\right) \frac{\partial}{\partial r^{\star}}\langle\bar{T}\rangle^{i 0}\right]=A \cdot d_{p}^{2} \quad \text { as } \quad r^{\star}<<\frac{R}{d_{p}} \\
& {\left[\left(\phi \lambda_{e f f}\right) \frac{\partial}{\partial r^{\star}}\left[a \log \left(r^{\star}\right)+b\right]\right]=A^{(1)} r^{\star}+B\left(R e_{p}\right)} \\
& \phi \lambda_{e f f}\left(r^{\star}\right)=A^{(2)} r^{\star 2}+B^{(1)}\left(R e_{p}\right) r^{\star} \\
& \phi \lambda_{e f f}\left(r^{\star}\right) \approx B^{(1)}\left(R e_{p}\right) r^{\star} \quad \text { as } \quad r^{\star}<<1
\end{aligned}
$$

where $r^{\star}=(R-r) / d_{p}$.

One can deduce that the effective conductivity is linear when the solid particles are considered to keep weak interactions with the wall boundary layer. It is consistent with the empirical correlation derived by [57]. The case of a strongly disturbed boundary layer is discussed in Appendix C

Finally, the effective conductivity in the entire medium reads, 


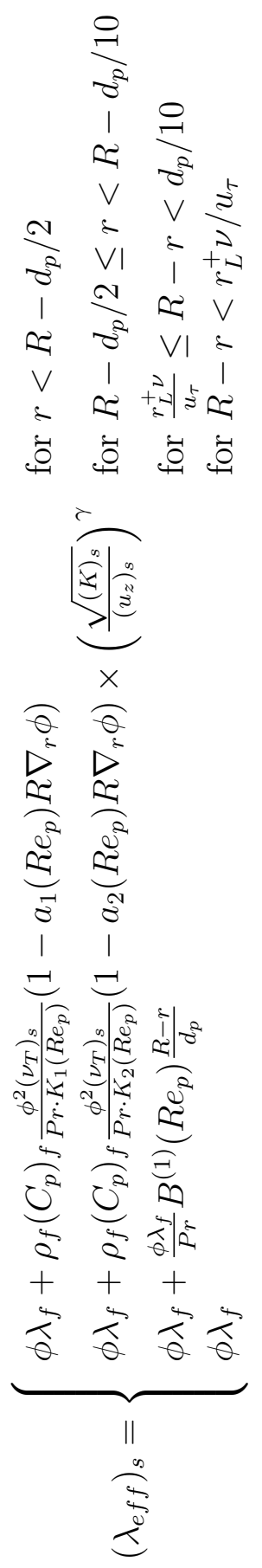




\begin{tabular}{|l|c|c|c|c|c|}
\hline$R e_{p}$ & $K_{1}$ & $K_{2}$ & $a_{1}$ & $a_{2}$ & $B^{(1)}$ \\
\hline 4000 & $2.71 \times 10^{-1}$ & $3.81 \times 10^{-2}$ & $5 \times 10^{-2}$ & $6.25 \times 10^{-2}$ & $8.55 \times 10^{-1}$ \\
\hline 7800 & $2.85 \times 10^{-1}$ & $3.69 \times 10^{-2}$ & $5.26 \times 10^{-2}$ & $6.07 \times 10^{-2}$ & 1.71 \\
\hline 15600 & $3.61 \times 10^{-1}$ & $3.59 \times 10^{-2}$ & $6.67 \times 10^{-2}$ & $5.88 \times 10^{-2}$ & 2.56 \\
\hline 19500 & $3.61 \times 10^{-1}$ & $3.39 \times 10^{-2}$ & $6.67 \times 10^{-2}$ & $5.56 \times 10^{-2}$ & 2.56 \\
\hline 23300 & $3.61 \times 10^{-1}$ & $3.39 \times 10^{-2}$ & $6.67 \times 10^{-2}$ & $5.56 \times 10^{-2}$ & 2.56 \\
\hline
\end{tabular}

Table 3: The macroscopic heat transfer coefficients values at different Reynolds numbers.

where $\left(\nu_{T}\right)_{s}$ is the dynamic viscosity defined by (22), the total kinetic energy $(K)_{s}$ and the axial velocity $\left(u_{z}\right)_{s}$ are obtained resolving the system of equations (20), (21), (28) and (34). Macorscopic thermal Prandtl numbers can be related to the molecular Prandtl number $\operatorname{Pr}, \sigma_{t 1}\left(R e_{p}\right)=$ $\operatorname{Pr} \cdot K_{1}\left(R e_{p}\right) / \phi$ and $\sigma_{t 2}\left(R e_{p}\right)=\operatorname{Pr} \cdot K_{2}\left(R e_{p}\right) / \phi$ with $\operatorname{Pr}=0.72$ for the considered fluid. Finally, $\gamma=0.5$ and $r_{L}^{+}=3$. The other coefficients values are reported in Table 3. It is worth noting that all coefficients tend to a constant value at high Reynolds, showing that the heat transfer mechanisms reach an equilibrium at high Reynolds.

\subsection{Model validation}

Macroscopic simulations are performed with the following boundary conditions: a constant heat flux at the wall, inlet and outlet boundaries are linked as periodic imposing a mass flow, then the overall heat flux brought at the tube wall is removed at the outlet $\left(\dot{m} C_{p}\left[T_{\text {bulk,exit }}-T_{\text {bulk,inlet }}\right]\right)$. The near wall mesh is fine enough to keep $y^{+} \approx 1$.

The radial profile of the effective thermal conductivity is well recovered and very good agreements are found in the near wall region where the temperature gradients are huge (Fig. 23). Thus, the radial profile of the temperature field matches well with the up-scaled pore scale reference data (Fig. 24). The wall temperature is also assessed with satisfaction (Table 4). The wall heat flux coefficient is then computed and very good agreements are found between the 1D macroscopic model and the REV-averaged 3D pore scale simulations (Tables 5 and 6).

From the simulation data a correlation for the wall heat transfer can be deduced (Fig. 25):

$$
N u_{w}=\frac{Q d_{p}}{\lambda_{f}\left(T_{w}-T_{b}\right)}=0.1 R e_{p}^{0.81}
$$




\begin{tabular}{|l|c|c|c|}
\hline$R e_{p}$ & $T_{w}$ micro $(\mathrm{K})$ & $T_{w}$ macro $(\mathrm{K})$ & $\left|\Delta T_{w}\right|(\mathrm{K})$ \\
\hline 4000 & 1333.59 & 1334.724 & 1.134 \\
\hline 7800 & 1090.04 & 1091.4664 & 1.426 \\
\hline 15600 & 968.54 & 961.153 & 7.39 \\
\hline 19500 & 942.655 & 944.04 & 1.39 \\
\hline 23300 & 924.75 & 932.47 & 7.72 \\
\hline
\end{tabular}

Table 4: Wall temperature, Micro vs. Macro.

\begin{tabular}{|l|c|c|c|}
\hline$R e_{p}$ & $T_{b}$ micro $(\mathrm{K})$ & $T_{b}$ macro $(\mathrm{K})$ & $\left|\Delta T_{b}\right|(\mathrm{K})$ \\
\hline 4000 & 829.46 & 822.87 & 6.59 \\
\hline 7800 & 813.88 & 811.96 & 1.92 \\
\hline 15600 & 806.55 & 805.35 & 1.2 \\
\hline 19500 & 805.22 & 804.14 & 1.08 \\
\hline 23300 & 804.18 & 801.3 & 2.88 \\
\hline
\end{tabular}

Table 5: Bulk temperature, Micro vs. Macro.

with the bulk temperature $T_{b}=\frac{\int_{0}^{R} r \phi\left\langle\bar{u}_{z}\right\rangle^{i}\langle\bar{T}\rangle^{i} d r}{\int_{0}^{R} r \phi\left\langle\bar{u}_{z}\right\rangle^{i} d r}$.

The correlation found in the present study agrees well with the correlations one can find in the literature. As it can be seen in the figure 26, the present correlation's trend and the range of the wall heat transfer coefficient values are consistent with those existing in the literature. Indeed, the slope given by the present correlation is very close to the one reported by [4]. It is worth noting that correlations, except the one presented in the present study and the one derived in [58], consider the temperature jump between the wall temperature and the fluid temperature at the wall $T_{w}-T(r=R)$ $\left(N u_{w}=\frac{Q d_{p}}{\lambda_{f}\left(T_{w}-T(r=R)\right)}\right)$. Consequently, the range of values for the wall heat transfer coefficient found in this study has to be compared to the correlation derived in [58]. One can thus be satisfied by the agreement found between the two of them. 


\begin{tabular}{|l|c|c|c|}
\hline$R e_{p}$ & $N u_{w}$ micro & $N u_{w}$ macro & $\left|\Delta N u_{w}\right|$ \\
\hline 4000 & 78.84 & 77.65 & $1.5 \%$ \\
\hline 7600 & 143.92 & 142.2 & $1.2 \%$ \\
\hline 15600 & 245.35 & 255.09 & $3.98 \%$ \\
\hline 19500 & 289.18 & 284.09 & $1.76 \%$ \\
\hline 23300 & 329.63 & 303 & $8.08 \%$ \\
\hline
\end{tabular}

Table 6: Evaluation of the wall heat transfer coefficient $N u_{w}=\frac{Q d_{p}}{\lambda_{f}\left(T_{w}-T_{b}\right)}$.

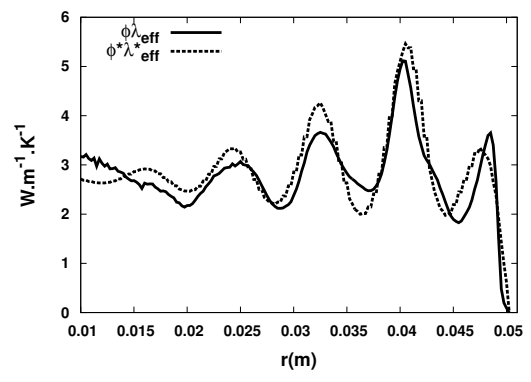

(a) $R e_{p}=4000$

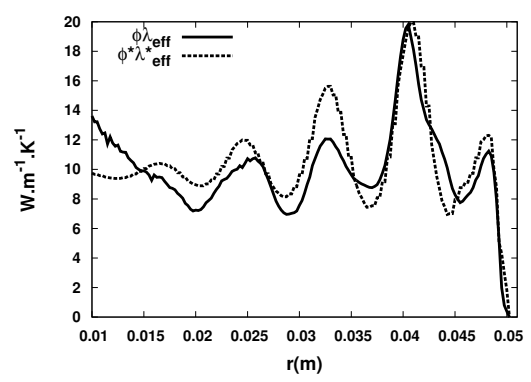

(c) $R e_{p}=15600$

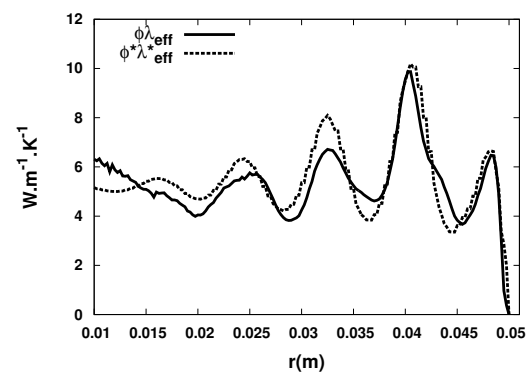

(b) $R e_{p}=7800$

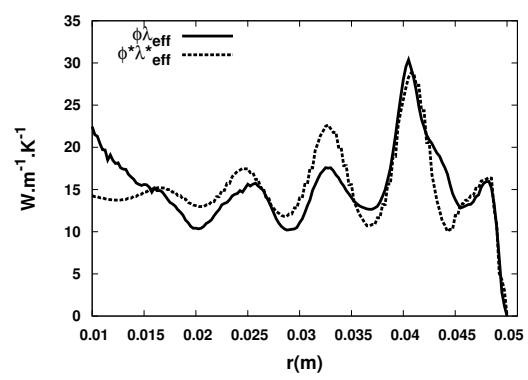

(d) $R e_{p}=23300$

Figure 23: Radial profile of the effective thermal conductivity, Micro $\phi \lambda_{\text {eff }}$ vs. Macro $\phi^{*} \lambda_{e f f}^{*}$. 


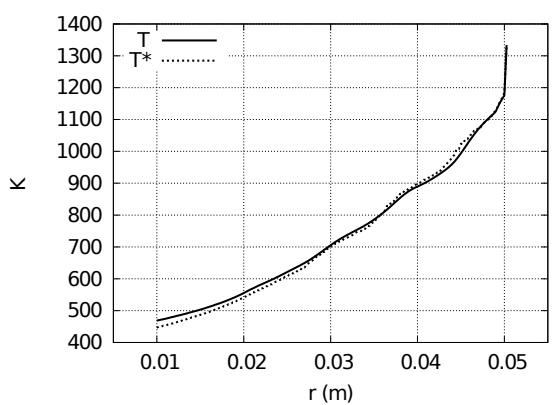

(a) $R e_{p}=4000$

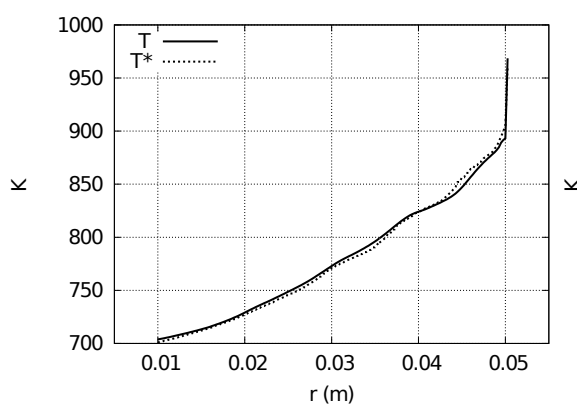

(c) $R e_{p}=15600$

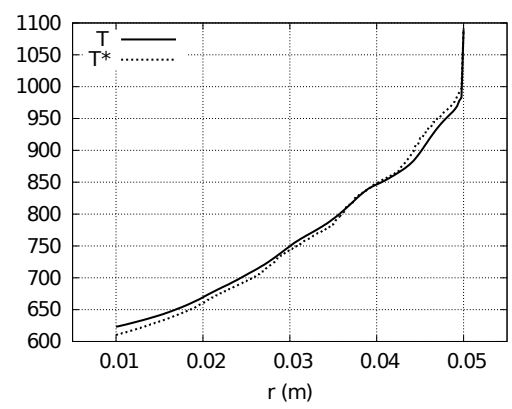

(b) $R e_{p}=7800$

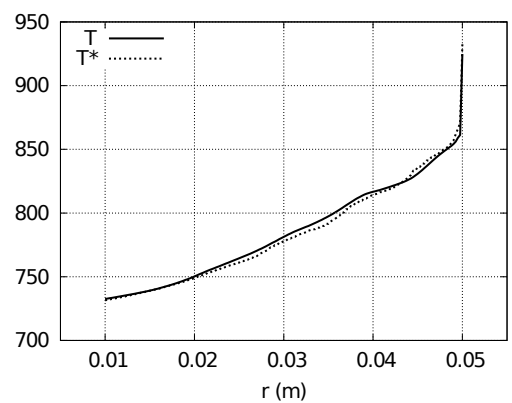

(d) $R e_{p}=23300$

Figure 24: Radial profile of the temperature field at the macroscopic scale, Micro $T$ vs Macro $T^{*}$

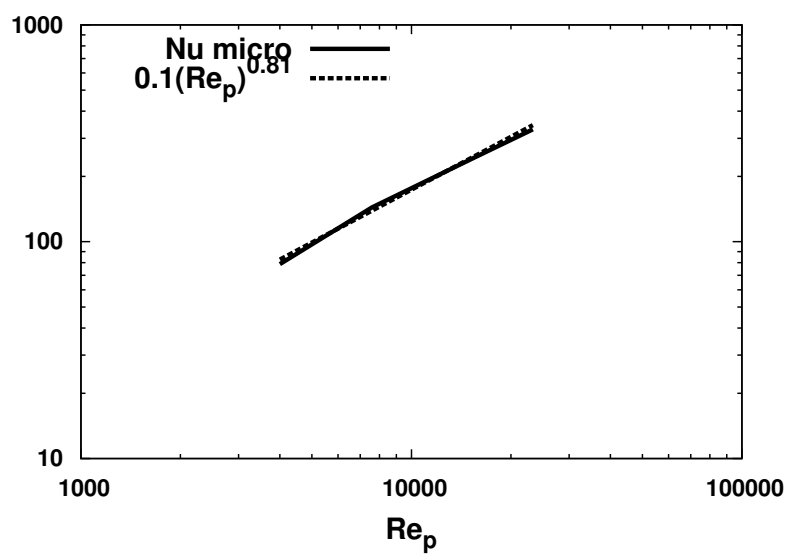

Figure 25: Correlation for the Nusselt number. 


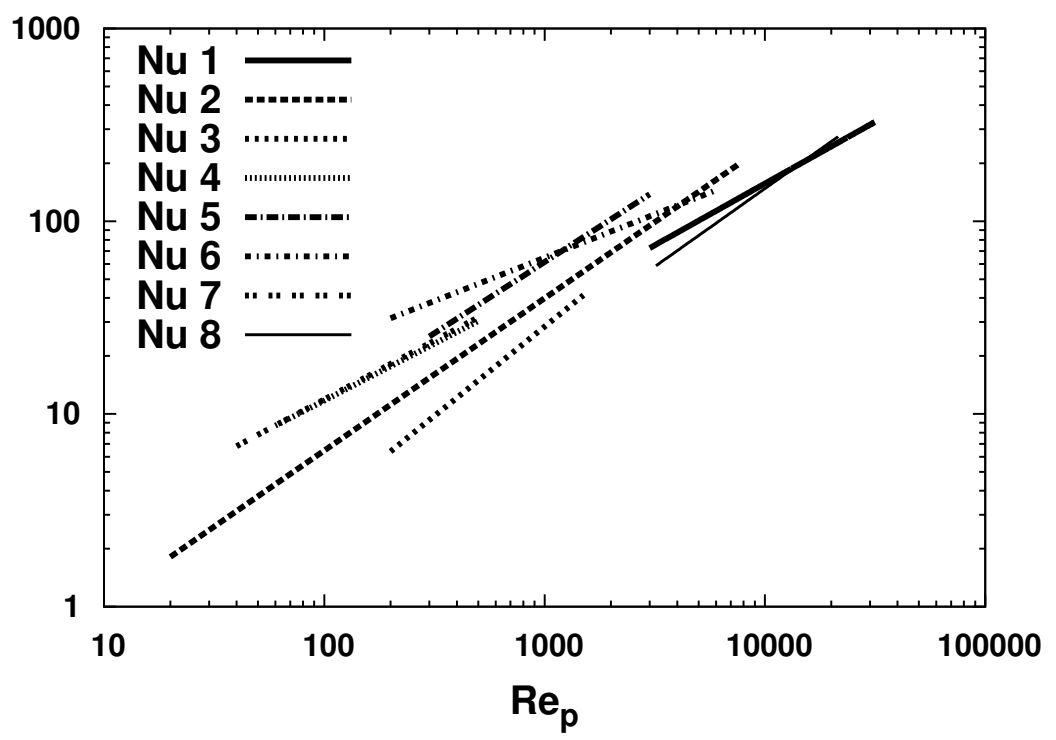

Figure 26: Correlations for the wall heat transfer coefficient. Nu1:[26]; Nu2: [4]; Nu3: [58]; Nu4: [59], Nu5: [60]; Nu6: 61]; Nu7: 62]; Nu8: Correlation derived in this study. 


\section{Conclusion} reads,

$$
\frac{\partial k}{\partial t}+\nabla_{j} k \overline{u_{j}}=-\nabla_{j}\left[\frac{\overline{P^{\prime} u_{j}^{\prime}}}{\rho}+\overline{u_{i}^{\prime} u_{i}^{\prime} u_{j}^{\prime}}\right]+\nu \nabla_{j}^{2} k-\overline{u_{i}^{\prime} u_{j}^{\prime}} \frac{\partial \overline{u_{i}}}{\partial x_{j}}-\epsilon
$$


Applying the volume averaging operator to A.1, one gets,

$$
\begin{aligned}
& \phi \frac{\partial\langle k\rangle^{i}}{\partial t}=-\nabla_{j}\left[\phi\left\langle\bar{u}_{j}\right\rangle^{i}\langle k\rangle^{i}\right]+\nu \nabla_{j}^{2}\left[\phi\langle k\rangle^{i}\right]-\left\langle R_{i j}\right\rangle^{i} \nabla_{j}\left[\phi\left\langle\bar{u}_{i}\right\rangle^{i}\right] \\
& -\nabla_{j}\left(\phi\left\langle\left[\frac{\overline{P^{\prime} u_{j}^{\prime}}}{\rho}+\overline{u_{i}^{\prime} u_{i}^{\prime} u_{j}^{\prime}}\right]\right\rangle^{i}\right)-\phi\langle\epsilon\rangle^{i} \\
& -\phi\left\langle\delta R_{i j} \nabla_{j} \delta \bar{u}_{i}\right\rangle^{i}+\frac{\nu}{V} \int \nabla_{j} k n_{j} d S-\nabla_{j}\left[\phi\left\langle\delta \overline{u_{j}} \delta k\right\rangle^{i}\right]
\end{aligned}
$$

where $\overline{u_{i}^{\prime} u_{j}^{\prime}}=R_{i j}$.

\section{Appendix B. dispersive kinetic energy}

Subtracting (7) from the pore scale momentum conservation equation and multiplying by $\delta \bar{u}_{i}$ the obtained equation and finally applying the volume averaging operator, one gets

$$
\begin{aligned}
0 & =-\left\langle\delta \overline{u_{i}} \nabla_{j}\left[\left\langle\overline{u_{i}}\right\rangle^{i} \delta \overline{u_{j}}\right]\right\rangle^{v}-\left\langle\delta \overline{u_{i}} \nabla_{j}\left[\left\langle\overline{u_{j}}\right\rangle^{i} \delta \overline{u_{i}}\right]\right\rangle^{v}-\left\langle\delta \overline{u_{i}} \nabla_{i} \frac{\delta \bar{P}}{\rho}\right\rangle^{v} \\
& +\nu\left\langle\delta \overline{u_{i}} \nabla_{j}^{2} \delta \overline{u_{i}}\right\rangle^{v}-\left\langle\delta \overline{u_{i}} \nabla_{j}\left[\delta \overline{u_{i}} \delta \overline{u_{j}}\right]\right\rangle^{v}-\left\langle\delta \overline{u_{i}} \nabla_{j} \delta R_{i j}\right\rangle^{v}
\end{aligned}
$$

which can be rearranged to obtain the governing equation for the kinetic energy of the dispersive fluctuations.

$$
\begin{aligned}
0 & =-\nabla_{j}\left[\phi\left\langle\overline{u_{j}}\right\rangle^{i}\left\langle k_{d}\right\rangle^{i}\right]-\frac{1}{\rho} \nabla_{i}\left[\phi\left\langle\delta \overline{u_{i}} \delta \bar{P}\right\rangle^{i}\right]-\nabla_{j}\left[\phi\left\langle\delta \overline{u_{i}} \delta \overline{u_{i}} \delta \overline{u_{j}}\right\rangle^{i}\right] \\
& +\nu \nabla_{j}^{2}\left[\phi\left\langle k_{d}\right\rangle^{i}\right]-\phi\left\langle\epsilon_{d}\right\rangle^{i}-\left\langle\delta \overline{u_{i}} \delta \overline{u_{j}}\right\rangle^{i} \nabla_{j}\left[\phi\left\langle\overline{u_{i}}\right\rangle^{i}\right]+\frac{\left\langle\overline{u_{i}}\right\rangle^{i}}{\rho V} \int \delta \bar{P} n_{i} d S \\
& -\frac{\nu\left\langle\overline{u_{i}}\right\rangle^{i}}{V} \int \nabla_{j} \delta \overline{u_{i}} n_{j} d S+\left\langle\overline{u_{j}}\right\rangle^{i} \nabla_{j} \phi\left[3\left\langle k_{d}\right\rangle^{i}-\frac{\left\langle\overline{u_{i}}\right\rangle^{i 2}}{2}\right] \\
& -\nu \nabla_{j}\left[\nabla_{j} \phi \frac{\left\langle\overline{u_{i}}\right\rangle^{i 2}}{2}\right]+\phi\left\langle\delta \overline{u_{i}} \delta \overline{u_{j}} \nabla_{j} \delta \overline{u_{i}}\right\rangle^{i}+\phi\left\langle\delta R_{i j} \nabla_{j} \delta \overline{u_{i}}\right\rangle^{i} \\
& -\nabla_{j}\left[\phi\left\langle\delta \overline{u_{i}} \delta R_{i j}\right\rangle^{i}\right]+\left[\left\langle R_{i j}\right\rangle^{i}+\left\langle\delta \overline{u_{i}} \delta \overline{u_{j}}\right\rangle^{i}\right]\left\langle\overline{u_{i}}\right\rangle^{i} \nabla_{j} \phi
\end{aligned}
$$

\section{Appendix C. Strongly disrupted boundary layer}

In this case, the temperature field can be split up according to

$$
\langle\bar{T}\rangle^{i}=\langle\bar{T}\rangle^{i 0}+\frac{\langle\bar{T}\rangle^{i 1}}{\epsilon}
$$


with $\epsilon<<1$. Thus,

$$
\begin{aligned}
& \frac{\partial}{\partial r^{\star}}\left[\left(\phi \lambda_{e f f}\right) \frac{\partial}{\partial r^{\star}}\langle\bar{T}\rangle^{i}\right]=A^{(3)} \\
& \frac{\partial}{\partial r^{\star}}\left[\left(\phi \lambda_{e f f}\right) \frac{\partial}{\partial r^{\star}}\left(\epsilon\langle\bar{T}\rangle^{i 0}+\langle\bar{T}\rangle^{i 1}\right)\right]=\epsilon A^{(3)}
\end{aligned}
$$

0 order term in $\epsilon$ :

$$
\frac{\partial}{\partial r^{\star}}\left[\left(\phi \lambda_{e f f}\right) \frac{\partial}{\partial r^{\star}}\langle\bar{T}\rangle^{i 1}\right]=0
$$

[6] T. Eppinger, K. Seidler, M. Kraume, Dem-cfd simulations of fixed bed
reactors with small tube to particle diameter ratios, Chem. Eng. J. 166
(2011) 324-331.

[6] T. Eppinger, K. Seidler, M. Kraume, Dem-cfd simulations of fixed bed
reactors with small tube to particle diameter ratios, Chem. Eng. J. 166
(2011) 324-331.

[6] T. Eppinger, K. Seidler, M. Kraume, Dem-cfd simulations of fixed bed
reactors with small tube to particle diameter ratios, Chem. Eng. J. 166
(2011) 324-331.

$$
\langle\bar{T}\rangle^{i 1}=\eta\left(R e_{p}\right) \nabla_{r}\langle\bar{T}\rangle^{i 0}
$$

Tacking into account the closure (C.4) in (C.3) one can deduce,

$$
\phi \lambda_{e f f}\left(r^{\star}\right)=\eta^{(1)}\left(R e_{p}\right) r^{\star 2}
$$

The profile is hence showed to be quadratic within a highly disturbed boundary layer. It is consistent with the empirical expression derived in [10].

\section{References}

[1] A. P. D. Wasch, G. F. Froment, Heat transfer in packed beds, Chem. Eng. Sci. 27 (1972) 567-576.

[2] G. Froment, Analysis and design of fixed bed catalytic reactors, Chem. Reaction Eng. 109 (1972) 1-55.

[3] J. Beek, Design of packed catalytic reactors, Adv. Chem. Eng. 3 (1962) 203-271.

[4] C. Li, B. Finlayson, Heat transfer in packed beds - a reevaluation, Chem. Eng. Sci. 32 (1977) 1055-1066.

[5] O. Bey, G. Eigenberger, Fluid flow through catalyst filled tubes, Chem Eng. Sci. 52 (1997) 1365-1376. 
[7] M. Behnam, A. Dixon, M. Nijemeisland, E. Stitt, A new approach to fixed bed radial heat transfer modeling using velocity fields from computational fluid dynamics simulations, Ind. Eng. Chem. Res. 52 (2013) $15244-15261$.

[8] A. Dixon, Fixed bed catalytic reactor modelling - the radial heat transfer problem, Canadian J. Chem. Eng. 90 (2012) 507-527.

[9] J. Papageorgiou, G. Froment, Simulation models accounting for radial voidage profiles in fixed bed reactors, Chem Eng. Sci. 50 (1995) 30433056 .

[10] M. Winterberg, E. Tsotsas, A simple and coherent set of coefficients for modelling of heat and mass transport with and without chemical reaction in tubes filled with spheres, Chem. Eng. Sci. 55 (2000) 967979.

[11] M. Winterberg, E. Tsotsas, Correlations for effective heat transport coefficients in beds packed with cylindrical particles, Chem Eng. Sci. 55 (2000) 5937-5943.

[12] D. Vortmeyer, E. Haidegger, Discrimination of three approaches to evaluate heat fluxes for wall-cooled fixed bed chemical reactors, Chem. Eng. Sci. 46 (1991) 2951-2660.

[13] E. Smirnov, A. Muzykantov, V. Kuzmin, A. Kronberg, I. Zolotarskii, Radial heat transfer in packed beds of spheres, cylinders and rashing rings. verification of model with a linear variation of $\lambda_{e r}$ in the vicinity of the wall, Chem Eng. J. 91 (2003) 243-248.

[14] D. Bunnell, H. Irvin, R. Olson, J. Smith, Effective thermal conductivities in gas-solid systems, Ind. Eng. Chem. 41 (1949) 1977-1981.

[15] S. Kwong, J. Smith, Radial heat transfer in packed beds, Ind. Eng. Chem. 49 (1957) 894-903.

[16] M. Ahmed, R. Fahien, Tubular reactor design-i, Chem. Eng. Sci. 35 (1980) 889-895.

[17] D. Gunn, M. Ahmed, The characterisation of radial heat transfer in fixed beds, IChemE Symp. Ser. 86 (1984) 513-520.

345 [18] D. Gunn, M. Ahmed, M. Sabri, Radial heat transfer to fixed beds of particles, Chem. Eng. Sci. 42 (1987) 2163-2171. 
[19] D. Gunn, M. Sabri, A distributed model for liquid-phase heat transfer in fixed beds, Int. J. Heat Mass Transfer 30 (1987) 1693-1702.

[20] J. Borkink, K. Westerterp, Significance of the radial porosity profile for the description of heat transport in wall-cooled packed beds, Chem. Eng. Sci. 49 (1994) 863-876.

[21] F. Mathey, Numerical up-scaling approach for the simulation of heattransfer in randomly packed beds, Int. J. Heat Mass Transfer 61 (2013) 451-463.

[22] J. Lerou, G. Froment, Velocity, temperature and conversion profiles in fixed bed catalytic reactors, Chem. Eng. Sci. 32 (1977) 853-861.

[23] J. Marivoet, P. Teodoriou, S. Wajc, Porosity, velocity and temperature profiles in cylindrical packed beds, Chem. Eng. Sci. 29 (1974) 1836-1840.

[24] D. Vortmeyer, J. Schuster, Evaluation of steady flow profiles in rectangular and circular packed beds by a variational method, Chem. Eng. Sci. 38 (1983) 1691-1699.

[25] O. Kalthoff, D. Vortmeyer, Ignition/extinction phenomena in a wall cooled fixed bed reactor. experiments and model calculations including radial porosity and velocity distributions, Chem. Eng. Sci. 35 (1980) $1637-1643$.

[26] I. Thiagalingam, I. Bennaceur, M. Dallet, S. Cadalen, P. Sagaut, Exact non local expression for the wall heat transfer coefficient for in tubular catalytic reactors, Int. J. Heat Fluid Flow 54 97-106.

[27] X. Jia, M. Gan, R. Williams, D. Rhodes, Validation of a digital packing algorithm in predicting powder packing densities, Powder Technology 174 (2007) 10-13.

[28] R. Caulkin, A.Ahmad, M. Fairweather, X. Jia, R. Williams, Digital prediction of complex cylinder packed columns, Comp. and Chem. Eng. 33 (2009) 10-21.

[29] R. Caulkin, M. Fairweather, X. Jia, R. Williams, A numerical case study of packed columns, in: European Symposium on Computer Aided process Engineering, Elsevier Science B.V., 2005.

[30] X. Jia, R. Williams, A packing algorithm for particles of arbitrary shapes, Powder Technology 120 (2001) 175-186. 
[41] W. Gray, P. Lee, On the theorems for local volume averaging of multiphase systems, Int. J. Multiphase Flow 3 (1977) 333-340.

[42] A. Nakayama, F. Kuwahara, A macroscopic turbulence model for flow in a porous medium, J. Fluids Eng. 121 (1999) 427-433. 
[43] M. Chandesris, G. Serre, P. Sagaut, A macroscopic turbulence model for flow in porous media suited for channel, pipe and rod bundle flows, Int. J. Heat Mass Transfer 49 (2006) 2739-2750.

[44] H. Mickeley, K. Smith, E. Korchak, Fluid flow in packed beds, Chem. Eng. Sci. 23 (1965) 237-246.

[45] I. Macdonald, M. El-Sayed, K. Mow, F. Dullien, Flow through porous media- ergun equation revisited, Ind. Eng. Chem. Fund. 18 (1979) 199208.

[46] D. van der Merwe, W. Gauvin, Velocity and turbulence measurements of air flow through packed bed, A.I.Ch.E. J. 17 (1971) 519-528.

[47] A. Dybbs, R. Edwards, A new look at porous media fluid mechanicsdarcy to turbulent, Fund. Trans. Phenom. Porous Media 82 (1984) 199256.

[48] T. Masuoka, Y. Takatsu, Turbulence model for flow through porous media, Int. J. Heat Mass Trans. 39 (1996) 2803-2809.

[49] M. de Lemos, M. Pedras, Recent mathematical models for turbulent flow in saturated rigid porous media, J. Fluids Eng. 123 (2002) 935-940.

[50] M. Pedras, M. de Lemos, Macroscopic turbulence modeling for incompressible flow through undeformable porous media, Int. J. Heat Mass Transfer 44 (2001) 1081-1093.

[51] F. Teruel, Rizwan-uddin, A new turbulence model for porous media flows. part i: Constitutive equations and model closure, Int. J. Heat Mass Trans. 52 (2009) 4264-4272.

[52] F. Teruel, Rizwan-uddin, A new turbulence model for porous media flows. part ii: Analysis and validation using microscopic simulations, Int. J. Heat Mass Trans. 52 (2009) 5193-5203.

[53] F. Teruel, Rizwan-uddin, Numerical computation of macroscopic turbulence quantities in representative elementary volume of the porous medium, Int. J. Heat Mass Trans. 53 (2010) 5190-5198.

[54] F. Pinson, O. Gregoire, O. Simonin, k-e macro-scale modeling of turbulence based on a two scale analysis in porous media, Int. J. Heat Fluid Flow 27 (2006) 955-966. 
[55] S.Ergun, Fluid flow through packed columns, Chem. Eng. Prog. 48 (1952) 89-94.

[56] F. Mathey, Macroscopic turbulent models for heat and mass transfer in catalyst reactors, in: AIP Conf. Proc. 1453, 2012.

450 [57] P. Cheng, D. Vortmeyer, Transverse thermal dispersion and wall channeling in a packed bed with forced convective flow, Chem. Eng. Sci. 43 (1988) 2523-2532.

[58] Y. Demirel, R. Sharma, H. Al-Ali, On the effective heat transfer parameters in a packed bed, Int. J. Heat Mass Trans. 43 (2000) 327-332.

[59] A. Dixon, M. DiCostanzo, B. Soucy, Fluid-phase radial transport in packed beds of low tube-to-particle diameter ratio, Int. J. Heat and Mass Transfer 27 (1984) 1701-1713.

[60] A. Dixon, Heat transfer in fixed beds at very low $(<4)$ tube-to-particle diameter ratio, Ind. Eng. Res. 36 (1997) 3053-3064.

[61] P. Peters, R. Schiffino, P. Harriott, Heat transfer in packed tube reactors, Int. Eng. Chem. Res. 27 (1988) 226-233.

[62] A. Dixon, L. Labua, Wall-to-fluid coefficients for fixed bed heat and mass transfer, Int. J. Heat Mass Transfer 28 (1985) 879-881.

[63] M. Drouin, O. Grégoire, O. Simonin, A. Chanoine, Macroscopic modeling of thermal dispersion for turblent flows in channels, Int. J. Heat Mass Transfer 53 (2010) 2206-2217. 